This document must be cited according to its final version which is published in a journal as:

H. Hammouri ${ }^{1}$ T, B. Targui ${ }^{1}$, F. Armanet ${ }^{1}$

" High gain observer based on a triangular structure",

International Journal of Robust and Nonlinear Control 12, 6 (2002)

497-518

This final version may be found:

http://dx.doi.org/10.1002/rnc.638

All open archive documents of $\mathbf{H}$. Hammouri are available at: http://hal.archives-ouvertes.fr/HAMMOURI-HASSAN

The professional web page (Fr/En) of $\mathrm{H}$. Hammouri is: http://www.lagep.univ-lyon1.fr/signatures/hammouri.hassan

All open archive documents of $H$. Hammouri research group (SNLEP) are available at:

http://hal.archives-ouvertes.fr/SNLEP

http://www.tinyurl.com/SNLEP

1

Université de Lyon, Lyon, F-69003, France; Université Lyon 1;

CNRS UMR 5007 LAGEP (Laboratoire d'Automatique et de GEnie des Procédés),

43 bd du 11 novembre, 69100 Villeurbanne, France

Tel +33 (0) 472431845 - Fax +33 (0) 472431699

http://www-lagep.univ-lyon1.fr/ http://www.univ-lyon1.fr http://www.cnrs.fr 


\title{
HIGH GAIN OBSERVER BASED ON A TRIANGULAR STRUCTURE
}

\author{
HASSAN HAMMOURI, BOUBEKEUR TARGUI, \\ AND FREDERIC ARMANET \\ Laboratoire d'Automatique et de Génie des Procédés, UPRES-A Q 500\%, LAGEP, CPE-Lyon, \\ Bat. 308G, UCBL I, 69622 Villeurbanne cedex, France.
}

\begin{abstract}
SUMMARY
A high gain observer based on a triangular structure of nonlinear systems is proposed . An algorithm permitting to calculate a gain of the observer is given. This observer synthesis is then extended to a class of multi-output nonlinear systems which contains the model of binary distillation columns. Finally, we illustrate the performance of the estimator using numerical simulations of a methanol-ethanol distillation column.
\end{abstract}

KEY WORDS Nonlinear systems, observability, observer

\section{INTRODUCTION}

The knowledge of state variables is often required in order to apply the advanced concepts of control and diagnosis to practical applications. One way permitting to obtain such variables, consists of combining a priori knowledge about physical system with experimental data to provide on-line estimator (observer). This estimator is generally a dynamic system obtained from the nominal model by adding a correction term which is proportional to some output deviation. In other words, given a nominal model:

$$
\left\{\begin{array}{c}
\dot{x}(t)=f(x(t), u(t)) \\
y(t)=h(x(t))
\end{array}\right.
$$

The state $x(t)$ belongs to an open subset $V$ of $\mathcal{R}^{n}$, the input $u(t)$ belongs to a Borelian subset $U$ of $\mathcal{R}^{m}$ and the output $y(t) \in \mathcal{R}^{p}$. An observer for (1) is generally a dynamic system of the form:

$$
\left\{\begin{array}{c}
\dot{\hat{x}}(t)=f(\hat{x}(t), u(t))-k(t)(h(\hat{x}(t))-y(t)) \\
\dot{r}(t)=F(r(t), u(t), y(t), \hat{x}(t)) \\
k(t)=\varphi(r(t))
\end{array}\right.
$$

$r(t)$ and $k(t)$ are called indifferently the gain of the observer. For some particular systems, the gain $k(t)$ does not depend on the input (see for instance the Luenberger observer). For general nonlinear systems the observer's gain depend on the input. This comes from the fact that the observability concept generally depends on the inputs: given an input $u$ defined on some interval $[0, T]$, we say that $u$ render system (1) observable if for every two initial states $x \neq x^{\prime}$; there exist $t \in[0, T]$ such that $h\left(x_{u}(t)\right) \neq h\left(x_{u}^{\prime}(t)\right)$, where $x_{u}(t)$ and $x_{u}^{\prime}(t)$ are respectively the trajectories associated with $u$ and issued from the initial states $x, x^{\prime}$. Generally a nonlinear system may be observable for some input and unobservable for an other one. For more details, see ${ }^{7,9}$.

An interesting class of nonlinear systems consists of those which are observable for every input, called uniformly observable systems. For this class of nonlinear systems, we can design an observer whose gain does not depend on the inputs (see, $1,2,3,5,6,8,10$ ). For such systems a canonical (triangular) form is designed in order to design an observer. To ensure the mathematical convergence, a particular high gain is required. However, the 
use of large gain may generate the so-called the peak phenomena (overshoot problem), moreover the estimator becomes noise sensitive. Due to nonlinearity of the system, the choice of the gain which gives the best compromise between fast convergence, the noise rejection and the attenuation of the peak phenomena becomes a difficult task, and only simulations allow to determine a plausible gain.

This paper is organized as follows: in section 2, we extend the observer synthesis stated in ${ }^{5}$ and ${ }^{6}$ to a class of multi-output uniformly observable systems. In section 3 , we apply this theoretical result to a binary distillation column.

\section{HIGH GAIN OBSERVER}

Consider the following control affine nonlinear system:

$$
\left\{\begin{array}{c}
\dot{x}(t)=f(x(t), u(t))=f_{0}(x(t))+\sum_{i=1}^{m} u_{i}(t) f_{i}(x(t)) \\
y(t)=h(x(t))
\end{array}\right.
$$

where $u=\left(u_{1}, \ldots, u_{m}\right)$.

In the single output case $(p=1)$ and when $U=\mathcal{R}^{m}$, the authors in ${ }^{4}$ and ${ }^{5}$ have shown that if in addition system (2) is uniformly observable, then,

$$
\left(z_{1}, \ldots, z_{n}\right)=\left(h(x), L_{f_{0}}(h(x)), \ldots, L_{f_{0}}^{n-1}(h(x))\right)
$$

becomes a local system of coordinates (almost everywhere) in which, system (2) takes the following canonical form:

$$
\left\{\begin{array}{c}
\dot{z}(t)=A z(t)+F_{0}(z(t))+\sum_{i=1}^{m} u_{i}(t) F_{i}(z(t)) \\
y(t)=C z(t)
\end{array}\right.
$$

where $L_{f_{0}}$ denotes the Lie derivative, $A, C$ and the $F_{j}$ 's are given by:

$$
A=\left[\begin{array}{ccccc}
0 & 1 & 0 & & 0 \\
\vdots & & 1 & & \\
0 & & & \ddots & 1 \\
0 & \ldots & & 0 & 0
\end{array}\right] ; C=\left[\begin{array}{lllll}
1 & 0 & . & 0
\end{array}\right]
$$

$F_{0}=\left[0, \ldots, 0, F_{0 n}\right]^{T}$; for $1 \leq i \leq m, F_{i}=\left[F_{i 1}, \ldots, F_{i n}\right]^{T}$ and $F_{i j}=F_{i j}\left(z_{1}, . ., z_{j}\right)$.

Moreover, if the $F_{i}$ 's are global Lipschitz (or if the concerned trajectories of system (3) are bounded), then an exponential observer for system (3) takes the following form:

$$
\dot{\hat{z}}(t)=A \hat{z}(t)+F_{0}(\hat{z}(t))+\sum_{i=1}^{m} u_{i}(t) F_{i}(\hat{z}(t))-S_{\theta}^{-1} C^{T}(C \hat{z}(t)-y(t))
$$

where $S_{\theta}$ is the symmetric positive definite (S.P.D.) matrix satisfying:

$$
\theta S_{\theta}+A^{T} S_{\theta}+S_{\theta} A=C^{T} C .
$$

For single output non control affine nonlinear system (1), in ${ }^{6}$, the authors have shown that if a single output system (1) is uniformly observable then, a similar transformation as above, transforms the system into the following form: 


$$
\left\{\begin{array}{l}
\dot{z}_{1}(t)=F_{1}\left(z_{1}(t), z_{2}(t), u(t)\right) \\
\dot{z}_{2}(t)=F_{2}\left(z_{1}(t), z_{2}(t), z_{3}(t), u(t)\right) \\
\vdots \\
\dot{z}_{n-1}(t)=F_{n-1}\left(z_{1}(t), \ldots, z_{n}(t), u(t)\right) \\
\dot{z}_{n}(t)=F_{n}\left(z_{1}(t), \ldots, z_{n}(t), u(t)\right) \\
y(t)=C z(t)=z_{1}(t)
\end{array}\right.
$$

with, the additional condition:

$$
\text { C1) } \frac{\partial F_{i}}{\partial z_{i+1}}(z, u) \neq 0 ; \forall(z, u) .
$$

Now set $F=\left[F_{1}, \ldots, F_{n}\right]^{T}$, if the following assumption holds:

$\left.H_{1}\right)$

i) The $F$ is a global Lipschitz function:

$$
\left\|\frac{\partial F(u, z)}{\partial z}(u, z)\right\| \text { is uniformly bounded. }
$$

ii) $\exists \alpha>0$ s.t. $\forall(u, z) \in\left(U \times R^{n}\right)$ we have:

$$
\frac{\partial F_{i}(u, z)}{\partial z_{i+1}} \geq \alpha
$$

then, we can find a constant vector $K$, such that the following system:

$$
\dot{\hat{z}}=F(\hat{z}, u)+\Delta_{\theta} K(C \hat{z}-y)
$$

becomes an exponential observer for system (4), where:

$\Delta_{\theta}$ is the $(n \times n)$ diagonal matrix:

$$
\Delta_{\theta}=\operatorname{diag}\left(\theta, \theta^{2}, \ldots, \theta^{n}\right)
$$

\section{Remark 1}

In the case where only condition $C 1$ holds and that $U$ is a bounded connected set, and the trajectories of (4) lie into a compact set, then hypothesis $H_{1}$ can be omited (see Remark 2 below).

In what follows, we give a constructive algorithm permitting to calculate such gain $K$, and we extend this observer synthesis to a class of multi-output systems.

To do so, we need some preliminary results:

Consider the following $k \times k$ matrix,

$$
A_{k}(t)=\left[\begin{array}{ccccc}
0 & a_{1}(t) & 0 & & 0 \\
\vdots & & a_{2}(t) & & \\
0 & & & \ddots & a_{k-1}(t) \\
0 & \ldots & & 0 & 0
\end{array}\right]
$$


where, the $a_{i}$ 's may be unknown and satisfying the following constraint:

$\left.H_{2}\right) \forall t \geq 0, \alpha_{1} \leq a_{i}(t) \leq \alpha_{2}$, for some constants $\alpha_{1}, \alpha_{2}>0$.

Let $S_{k}$ be the $k \times k$ symmetric matrix of the form:

$$
S_{k}=\left[\begin{array}{ccccc}
s_{11} & s_{12} & 0 & & 0 \\
s_{12} & s_{22} & \ddots & & \vdots \\
0 & \ddots & & \ddots & 0 \\
\vdots & & & \ddots & s_{k-1 k} \\
0 & \ldots & 0 & s_{k-1 k} & s_{k k}
\end{array}\right]
$$

and denote by $C_{k}$ the k-row vector:

$$
C_{k}=[1,0, . ., 0]
$$

we then obtain the following:

\section{Lemma 1}

Assume that $H_{2}$ holds, then for every $\rho>0$, we can find $\eta>0$ and a symmetric positive definite (S.P.D.) matrix $S_{k}$ of the form (6) such that:

$$
\forall t \geq 0, A_{k}^{T}(t) S_{k}+S_{k} A_{k}(t)-\rho C_{k}^{T} C_{k} \leq-\eta I_{k} .
$$

Moreover $S_{k}$ depends only on the bounds $\alpha_{1}, \alpha_{2}$ and not on the knowledge of $A_{k}(t)$. The proof of lemma 1 will be given below.

Now, let $A_{n}(t)$ be the $n \times n$ matrix of the form (6) in which $a_{i}(t)$ is replaced by $\frac{\partial F_{i}}{\partial z_{i+1}}(\hat{z}(t)+$ $\omega(t), u(t))$, where $\omega(t)$ is any vector of $R^{n}$. Then from hypothesis $H_{1}$, it follows that the $a_{i}$ 's satisfy $H_{2}$, here, $\alpha_{1}=\alpha$ and $\alpha_{2}$ is the Lipschitz constant of $F$, given by sup $\left\|\frac{\partial F}{\partial z}(z(t), u(t))\right\|, \quad(z, u) \in R^{n} \times U$. We can state the following:

\section{Theorem 1.}

Assume that $H_{1}$ holds, then system (5) in which $K$ is replaced by $\Delta_{\theta} S_{n}^{-1} C^{T}$ is an exponential observer.

The proof is similar to this given in ${ }^{6}$ ( the extension of this result, is given in Theorem 2 below).

In what follows, we will extend this observer synthesis to the following class of nonlinear systems which contains the model of binary distillation columns:

$$
\left\{\begin{array}{l}
\dot{x}^{1}(t)=f^{1}(x(t), u(t))+d^{1}(t) \\
\dot{x}^{2}(t)=f^{2}(x(t), u(t))+d^{2}(t) \\
y(t)=\left(y_{1}(t), y_{2}(t)\right)^{T}=\left(C_{n_{1}} x^{1}(t), C_{n_{2}} x^{2}(t)\right)^{T}
\end{array}\right.
$$


where $x=\left[\begin{array}{c}x^{1} \\ x^{2}\end{array}\right] \in \mathcal{R}^{n} ; x^{i}=\left[\begin{array}{c}x_{1}^{i} \\ \vdots \\ x_{n_{i}}^{i}\end{array}\right] \in \mathcal{R}^{n_{i}}$ for $i=1,2\left(n=n_{1}+n_{2}\right) ; y_{i}=C_{n_{i}} x^{i}=x_{1}^{i}$ $\left(C_{n_{i}}=[1,0, \ldots, 0]\right) ; u$ is an known signal such that $\forall t, u(t) \in U$, the $d^{i}$ 's are unknown and bounded disturbances, with $\sup _{t \geq 0}\left\|d^{i}(t)\right\|=d<+\infty$.

Finally, the nonlinear dynamics satisfy the following triangular structure:

$$
f^{1}(x, u)=\left[\begin{array}{c}
f_{1}^{1}\left(x_{1}^{1}, x_{2}^{1}, u\right) \\
f_{2}^{1}\left(x_{1}^{1}, x_{2}^{1}, x_{3}^{1}, u\right) \\
\vdots \\
f_{n_{1}-1}^{1}\left(x^{1}, u\right) \\
f_{n_{1}}^{1}(x, u)
\end{array}\right] ; f^{2}(x, u)=\left[\begin{array}{c}
f_{1}^{2}\left(x_{1}^{2}, x_{2}^{2}, u\right) \\
f_{2}^{2}\left(x_{1}^{2}, x_{2}^{2}, x_{3}^{2}, u\right) \\
\vdots \\
f_{n_{2}-2}^{2}\left(x_{1}^{2}, \ldots, x_{n_{2}-1}^{2}, u\right) \\
f_{n_{2}-1}^{2}(x, u) \\
f_{n_{2}}^{2}(x, u)
\end{array}\right]
$$

The following lemma gives a sufficient condition which guarantee the uniform observability.

\section{Lemma 2}

If for every $(x, u) \in R^{n} \times U, \frac{\partial f_{j}^{i}}{\partial x_{j+1}^{i}}(x, u) \neq 0$; then system (8) is uniformly observable.

Proof. The uniform observability means that for every initial states $x \neq \bar{x}$ and every input from any $[0, T]$ into $U$, the associated output $y(t)=h(x(t))$ and $\bar{y}(t)=h(\bar{x}(t))$ are not identically equal on $[0, T]$, where $x(t)$ (respectively $\bar{x}(t)$ ) is the trajectory corresponding to the input $u$ and initial state $x$ (resp. $\bar{x}$ ).

To prove the Lemma 2, it suffices to show that if for every $t \in[0, T], h(x(t))=h(\bar{x}(t))$,

then $x=\bar{x}_{\dot{x}}\left[\begin{array}{l}x^{1} \\ x^{2}\end{array}\right]$ and $h(x)=\left[\begin{array}{l}x_{1}^{1} \\ x_{1}^{2}\end{array}\right]$.

Assume that $h(x(t))=h(\bar{x}(t)), \forall t \in[0, T]$.

We obtain

$$
x_{1}^{1}(t)=\bar{x}_{1}^{1}(t), \forall t \in[0, T] .
$$

Differentiating this last equality, we get:

$$
f_{1}^{1}\left(x_{1}^{1}(t), x_{2}^{1}(t), u(t)\right)=f_{1}^{1}\left(x_{1}^{1}(t), \bar{x}_{2}^{1}(t), u(t)\right)
$$

Using the mean value theorem, there exists a $\omega_{1}(t) \in[0,1]$ s.t.

$$
\frac{\partial f_{1}^{1}}{\partial x_{2}^{1}}\left(x_{1}^{1}(t), x_{2}^{1}(t)+\omega_{1}(t)\left(x_{2}^{1}(t)-\bar{x}_{2}^{1}(t)\right), u(t)\right)\left(x_{2}^{1}(t)-\bar{x}_{2}^{1}(t)\right)=0
$$

But

$$
\frac{\partial f_{1}^{1}}{\partial x_{2}^{1}}(x, u) \neq 0, \forall(x, u) \in R^{n} \times U
$$

Thus

$$
x_{2}^{1}(t)=\bar{x}_{2}^{1}(t), \forall t \in[0, T] .
$$

Using the triangular structure of $f^{1}$ and the fact that $\frac{\partial f_{i}^{1}}{\partial x_{i+1}}(x, u) \neq 0 ; \forall(x, u) \in R^{n} \times U$, an induction proof gives $x^{1}(t)=\bar{x}^{1}(t), \forall t \in[0, T]$. 
In similar manner, using the triangular structure of $f^{2}$ and the fact that $x^{1}(t)=\bar{x}^{1}(t)$, and $x_{1}^{2}(t)=\bar{x}_{1}^{2}(t)$ we can show by similar induction proof that $\forall t \geq 0, x^{2}(t)=\bar{x}^{2}(t)$. This ends the proof of the lemma 2 .

As in the single output case (see hypothesis $H_{1}$ ), the design of an observer for (8), requires the following assumption :

$\left.H_{3}\right)$

i) $\exists c>0 ; \forall(x, u),\left\|\frac{\partial f^{i}}{\partial x}(x, u)\right\| \leq c$

ii) $\exists \alpha>0 ; \forall x ; \forall u, \frac{\partial f_{j}^{i}}{\partial x_{j+1}^{i}}(x, u) \geq \alpha$, for $i=1,2$ and for $1 \leq j \leq n_{i}-1$.

\section{Remark 2}

If $U$ is a connected compact subset of $R^{m}$ and that the trajectories of system (8) lie into a connected compact subset $K$ of $R^{n}$, then hypothesis $H_{3}$ can be replaced by:

$$
\frac{\partial f^{i}}{\partial x_{j+1}^{i}}(x, u) \neq 0, \forall(x, u) \in(K \times U) .
$$

Proof of remark 2. Indeed, From $C 2$, it follows that $\exists \alpha>0 ; \forall(x, u) \in(K \times U)$ we have $\left|\frac{\partial f_{j}^{i}}{\partial x_{j+1}^{i}}(x, u)\right| \geq \alpha$.

Now, since $K \times U$ is a connected set, it follows that $\frac{\partial f_{j}^{i}}{\partial x_{j+1}^{i}}$ keeps a constant sign.

Using the simple change of coordinates $\tilde{x}_{j}^{i}=\varepsilon_{i j} x_{j}^{i}$ where $\varepsilon_{i j}=\operatorname{sign}\left(\frac{\partial f_{j}^{i}}{\partial x_{j+1}^{i}}\right)$, system (8) becomes:

$$
\left\{\begin{array}{l}
\dot{\tilde{x}}^{1}(t)=\tilde{f}^{1}(\tilde{x}(t), u(t))+\tilde{d}^{1}(t) \\
\dot{\tilde{x}}^{2}(t)=\tilde{f}^{2}(\tilde{x}(t), u(t))+\tilde{d}^{2}(t) \\
y(t)=\left(C_{n_{1}} \tilde{x}^{1}(t), C_{n_{2}} \tilde{x}^{2}(t)\right)^{T}
\end{array}\right.
$$

Moreover, it has a similar triangular structure (8) and,

$$
\frac{\partial \tilde{f}_{j}^{i}}{\partial \tilde{x}_{j+1}^{i}}(\tilde{x}, u) \geq \alpha, \forall(\tilde{x}, u) \in(\tilde{K} \times U)
$$

where $\tilde{K}=\left\{\tilde{x} / \tilde{x}_{j}^{i}=\varepsilon_{i j} x_{j}^{i}\right.$ and $\left.x \in K\right\}$.

Now as in ${ }^{5}$, we can always find global Lipschitz functions having similar triangular structure as $\breve{f}^{1}, \breve{f}^{2}$ and satisfying:

a) $\breve{f}^{i}(\tilde{x}, u)=\tilde{f}^{i}(\tilde{x}, u)$, for $i=1,2$ and for every $(\tilde{x}, u) \in(\tilde{K} \times U)$

b) $\frac{\partial \breve{f}_{j}^{i}}{\partial \tilde{x}_{j+1}^{i}}(\tilde{x}, u) \geq \alpha, \forall(\tilde{x}, u) \in\left(R^{n} \times U\right)$

This ends the proof of Remark 2 .

Noticing that if $U$ is not a connected set, then condition $H_{3}-i$ ) together with $C 2$ are not sufficient for the existence of an observer of constant gain.

\section{Conter-example}

Consider the following system: 


$$
(S)\left\{\begin{array}{l}
\dot{x}=\left[\begin{array}{ll}
0 & u \\
0 & 0
\end{array}\right] x \\
y(t)=x_{1}=C x
\end{array}\right.
$$

with $u \in U=\{+1,-1\}$.

Clearly system (S) has a triangular structure and satisfies $H_{3}-i$ ), and $C 2$.

However (S) cannot admit an observer with constant gain.

Proof of the Conter-example. Assuming that (S) admits an observer of constant gain:

$$
\dot{\hat{x}}=\left[\begin{array}{ll}
0 & u \\
0 & 0
\end{array}\right] \hat{x}+K(C \hat{x}-y)
$$

with $K=\left[\begin{array}{l}k_{1} \\ k_{2}\end{array}\right]$.

Thus the obtained error equation:

$$
\dot{e}=\left[\begin{array}{ll}
k_{1} & u \\
k_{2} & 0
\end{array}\right] e
$$

becomes simultaneous asymptotically stable for every input $u: R^{+} \rightarrow\{1,-1\}$. Now set $A_{1}=\left[\begin{array}{ll}k_{1} & 1 \\ k_{2} & 0\end{array}\right]$ and $A_{2}=\left[\begin{array}{cc}k_{1} & -1 \\ k_{2} & 0\end{array}\right]$, in particular the two systems:

$$
\dot{e}=A_{i} e \quad(i=1,2)
$$

becomes asymptotically stable.

This implies that the spectrums $S p\left(A_{i}\right)$ are in $\mathcal{C}=\{\lambda \in \mathcal{C}: \operatorname{Re}(\lambda)<0\}$.

Let us examine this last fact:

The respective characteristic polynomial of $A_{1}$ and $A_{2}$ are $P_{1}=\lambda^{2}-k_{1} \lambda-k_{2}$ and $P_{2}=\lambda^{2}-k_{1} \lambda+k_{2}$.

Set $r_{11}, r_{12}$ ( resp. $\left.r_{21}, r_{22}\right)$ the roots of $P_{1}$ (resp. $P_{2}$ ), we obtain:

$$
\left\{\begin{array}{l}
r_{11}+r_{12}=-k_{1} \\
r_{11} r_{12}=-k_{2} \\
r_{21}+r_{22}=-k_{1} \\
r_{21} r_{22}=k_{2}
\end{array}\right.
$$

Thus the stability of $A_{1}$ (resp. of $A_{2}$ ) is equivalent to $k_{1}<0$ and $k_{2}<0$ (resp. $k_{1}<0$ and $\left.k_{2}>0\right)$. Consequently, there exist no $k_{1}, k_{2}$ which give rise to both simultaneous asymptotic stability of $A_{1}$ and $A_{2}$.

Now, we can state our main result:

\section{Theorem 2.}

Let $\delta_{1}>0, \delta_{2}>0$ be two constants satisfying:

$$
\frac{2 n_{1}-1}{2 n_{2}-1} \delta_{1}<\delta_{2}<\frac{2 n_{1}+1}{2 n_{2}-1} \delta_{1}
$$


set, $\Delta_{\theta^{\delta_{i}}}=\operatorname{diag}\left(\theta^{\delta_{i}}, \theta^{2 \delta_{i}}, \ldots, \theta^{n_{i} \delta_{i}}\right),(i=1,2)$, and assume that $H_{3}$ holds for system (8). Then there exist S.P.D. matrices $S_{n_{i}}(i=1,2)$ of the form $(7)$ such that the following system:

$$
\left\{\begin{array}{l}
\dot{\hat{x}^{1}}(t)=f^{1}(\hat{x}(t), u(t))-r_{1} \Delta_{\theta^{\delta_{1}}} S_{n 1}^{-1} C_{n 1}^{T}\left(C_{n 1} \hat{x}(t)-y_{1}(t)\right) \\
\dot{\hat{x}}^{2}(t)=f^{2}(\hat{x}(t), u(t))-r_{2} \Delta_{\theta^{\delta_{2}}} S_{n 2}^{-1} C_{n 2}^{T}\left(C_{n 2} \hat{x}(t)-y_{2}(t)\right)
\end{array}\right.
$$

becomes an exponential estimator:

$\exists r_{1}, r_{2}>0 ; \exists \theta_{0}>0 ; \forall \theta \geq \theta_{0} ; \exists \lambda_{1}, \lambda_{2}>0 ; \exists \sigma>0$,such that for every $\hat{x}(0), x(0)$, we have: $\|\hat{x}(t)-x(t)\| \leq \lambda_{1} e^{-\sigma t}\|\hat{x}(0)-x(0)\|+\lambda_{2} d$, here $d$ is the upper bound of $\left\|d^{i}(t)\right\|, i=1,2$. This means that the ball $B\left(0, \lambda_{2} d\right)$ is exponentially asymptotically attracts the error of estimation.

\section{Remark 3}

Noticing that for $\theta$ large $\sigma$ becomes large and hence the speed of convergence of the observer may be chosed arbitrary . However, for $\theta$ sufficiently large, $\lambda_{1}$ may become large and hence the disturbance may affect the performance of the estimator. Consequently, a good choice of $\theta$ which not affect the performances of the estimator is necessary. Due to nonlinearity, this problem becomes very difficult and only simulations allows to obtain a compromise between the fast convergence of the observer and the disturbance sensitivity. First, let us give the proof of Lemme 1 .

Proof of Lemma 1.We will give the proof by induction.

For $k=2$; consider $A_{2}(t)=\left[\begin{array}{cc}0 & a_{1}(t) \\ 0 & 0\end{array}\right]$, with $0<\alpha_{1} \leq a_{1}(t) \leq \alpha_{2}, \forall t \geq 0$.

Let $S_{2}=\left[\begin{array}{ll}s_{11} & s_{12} \\ s_{12} & s_{22}\end{array}\right]$ be symmetric positive definite (S.P.D), and set;

$$
P_{2}(t)=A_{2}^{T}(t) S_{2}+S_{2} A_{2}(t)-\rho C_{2}^{T} C_{2}=\left[\begin{array}{cc}
-\rho & s_{11} a_{1}(t) \\
s_{11} a_{1}(t) & 2 s_{12} a_{1}(t)
\end{array}\right]
$$

for some $\rho>0$ and where $X_{2}=\left[x_{1}, x_{2}\right]^{T}$.

We have:

$$
X_{2}^{T} P_{2}(t) X_{2}=-\rho x_{1}^{2}+2 s_{11} a_{1}(t) x_{1} x_{2}+2 s_{12} a_{1}(t) x_{2}^{2}
$$

Now choosing $s_{11}>0, s_{22}>0$ and $s_{12}<0$, and using inequality $0<\alpha_{1} \leq a_{1}(t) \leq \alpha_{2}$, we obtain:

$$
X_{2}^{T} P_{2}(t) X_{2} \leq-\rho x_{1}^{2}+2 s_{11} \alpha_{2} x_{1} x_{2}-2 s_{12} \alpha_{1} x_{2}^{2}
$$

Now, choosing $s_{12}<0$ and $s_{22}>0$ such that:

$$
\begin{gathered}
\left|s_{12}\right|>\frac{s_{11}^{2} \alpha_{2}^{2}}{2 \rho \alpha_{1}} \\
s_{22}>\frac{s_{12}^{2}}{s_{11}}
\end{gathered}
$$

Inequality (12), implies that:

$$
X_{2}^{T} P_{2}(t) X_{2} \leq-\eta\left\|X_{2}\right\|^{2}, \text { where } \eta=\left(1-\frac{2 s_{11} \alpha_{2}}{2 \rho \alpha_{1}\left|s_{12}\right|}\right) \min \left\{\rho, 2\left|s_{12}\right| \alpha_{1}\right\}>0,
$$


and inequality (13) imposes that $S_{2}$ remains S.P.D. Hence lemma 1 is proved for $k=2$. Now assume that for every $\rho>0$; there exist $\eta_{k-1}>0$ and a S.P.D matrix $S_{k-1}$ of the form (7) such that:

$$
\forall t \geq 0, A_{k-1}^{T}(t) S_{k-1}+S_{k-1} A_{k-1}(t)-\rho C_{k-1}^{T} C_{k-1} \leq-\eta_{k-1} I_{k-1}
$$

Let us show that for any $\rho>0$; there exists $\eta>0$ and a S.P.D matrix $S_{k}$, such that inequality (14) holds for $S_{k}$ and $C_{k}$.

Set, $S_{k}=\left[\begin{array}{cc}S_{k-1} & F_{k-1} \\ F_{k-1}^{T} & s_{k k}\end{array}\right]$, where $S_{k-1}$ satisfies (15) and $F_{k-1}$ is a (k-1)-column vector of the form $F_{k-1}=\left[0, \ldots, 0, s_{k-1 k}\right]^{T}$.

Set,

$$
A_{k}(t)=\left[\begin{array}{cc}
A_{k-1} & v_{k-1}(t) \\
0 & 0
\end{array}\right]
$$

where $v_{k-1}$ is the $(\mathrm{k}-1)$-column vector $v_{k-1}=\left[0, \ldots, 0, a_{k-1}(t)\right]^{T}$.

Consider the symmetric matrix $P_{k}(t)=S_{k} A_{k}(t)+A_{k}(t)^{T} S_{k}-\rho C_{k}^{T} C_{k}$. As for $k=2$, we will show that we can choose $S_{k}$ such that for every $X_{k} \in \mathcal{R}^{k}, X_{k}^{T} P_{k}(t) X_{k} \leq-\eta\left\|X_{k}\right\|^{2}$. A simple computation gives:

$$
P_{k}(t)=\left[\begin{array}{cc}
P_{k-1}(t) & S_{k-1} v_{k-1}(t)+A_{k-1}^{T}(t) F_{k-1} \\
F_{k-1}^{T} A_{k-1}(t)+v_{k-1}^{T}(t) S_{k-1} & 2 F_{k-1}^{T} v_{k-1}(t)
\end{array}\right]
$$

hence,

$$
X_{k}^{T} P_{k}(t) X_{k}=X_{k-1}^{T} P_{k-1}(t) X_{k-1}+2 X_{k-1}^{T} S_{k-1} v_{k-1}(t) x_{k}+2 s_{k-1 k} a_{k-1}(t) x_{k}^{2} .
$$

Now, choosing $s_{k-1 k}<0$ and using the fact that $0<\alpha_{1} \leq a_{k-1}(t) \leq \alpha_{2}$, we get:

$$
X_{k}^{T} P_{k}(t) X_{k}=X_{k-1}^{T} P_{k-1}(t) X_{k-1}+2 \alpha_{2}\left\|S_{k-1}\right\|\left\|X_{k-1}\right\|\left|x_{k}\right|+2 s_{k-1 k} \alpha_{1} x_{k}^{2}
$$

Inequalities (14) and (15) yield to:

$$
X_{k}^{T} P_{k}(t) X_{k} \leq-\eta_{k-1}\left\|X_{k-1}\right\|^{2}+2 \alpha_{2}\left\|S_{k-1}\right\|\left\|X_{k-1}\right\|\left|x_{k}\right|+2 s_{k-1 k} \alpha_{1} x_{k}^{2}
$$

Now, consider $s_{k-1 k}<0$ and such that:

$$
\frac{\alpha_{2}\left\|S_{k-1}\right\|}{\sqrt{2\left|s_{k-1 k}\right| \alpha_{1} \eta_{k-1}}}<1
$$

or equivalently;

$$
s_{k-1 k}<-\frac{\alpha_{2}^{2}\left\|S_{k-1}\right\|^{2}}{2 \alpha_{1} \eta_{k-1}}
$$

Combining this last inequality with (16), we get:

$$
X_{k}^{T} P_{k}(t) X_{k} \leq-\eta\left\|X_{k}\right\|^{2}, \text { where } \eta=\left(1-\frac{\alpha_{2}\left\|S_{k-1}\right\|}{\sqrt{2\left|s_{k-1 k}\right| \alpha_{1} \eta_{k-1}}}\right) \min \left\{\eta_{k-1}, 2 s_{k-1 k} \alpha_{1}\right\}
$$

To end the proof, we will choose $s_{k k}$ so that $S_{k}$ remains S.P.D.

From the above notations, we have:

$$
\begin{aligned}
X_{k}^{T} S_{k} X_{k} & =X_{k-1}^{T} S_{k-1} X_{k-1}+2 F_{k-1}^{T} X_{k-1} x_{k}+s_{k k} x_{k}^{2} \\
& \geq X_{k-1}^{T} S_{k-1} X_{k-1}-2\left\|F_{k-1}\right\|\left\|X_{k-1}\right\|\left|x_{k}\right|+s_{k k} x_{k}^{2} \\
& \geq X_{k-1}^{T} S_{k-1} X_{k-1}-2\left|s_{k-1 k}\right|\left\|X_{k-1}\right\|\left|x_{k}\right|+s_{k k} x_{k}^{2}\left(\text { since }\left\|F_{k-1}\right\|=\left|s_{k-1 k}\right|\right)
\end{aligned}
$$


From the induction hypothesis, we know that $S_{k-1}$ is S.P.D. Hence there exist a constant $\tilde{\nu}_{k-1}>0$ s.t. $X_{k-1}^{T} S_{k-1} X_{k-1} \geq \tilde{\nu}_{k-1}\left\|X_{k-1}\right\|^{2}$.

Thus,

$$
X_{k}^{T} S_{k} X_{k} \geq \tilde{\nu}_{k-1}\left\|X_{k-1}\right\|^{2}-2\left|s_{k-1 k}\right|\left\|X_{k-1}\right\|\left|x_{k}\right|+s_{k k} x_{k}^{2}
$$

Now, choosing $s_{k k}$ such that

$$
s_{k k}>\frac{s_{k-1 k}^{2}}{\nu_{k-1}}
$$

or equivalently;

$$
\frac{\left|s_{k-1 k}\right|}{\sqrt{s_{k k} \nu_{k-1}}}<1
$$

we obtain:

$$
X_{k}^{T} S_{k} X_{k} \geq \nu_{k}\left\|X_{k}\right\|^{2} \text { where } \nu_{k}=\left(1-\frac{\left|s_{k-1 k}\right|}{\sqrt{s_{k k} \nu_{k-1}}}\right) \inf \left\{\tilde{\nu}_{k-1}, s_{k k}\right\}>0
$$

This ends the proof of lemma 1.

proof of Theorem 2. Let $\alpha, c$ be the positive constants given in assumption $H_{3}$ and consider two any unknown matrices:

$$
A_{n_{i}}(t)=\left[\begin{array}{ccccc}
0 & a_{1}^{i}(t) & 0 & \ldots & 0 \\
\vdots & & a_{2}^{i}(t) & & \vdots \\
0 & & & \ddots & a_{n_{i}-1}^{i}(t) \\
0 & \ldots & & 0 & 0
\end{array}\right] \text { for } i=1,2
$$

such that : $\alpha \leq a_{j}^{i}(t) \leq c$, for $i=1,2$ and $1 \leq j \leq n_{i}$.

From lemma 1 in which $\alpha_{1}, \alpha_{2}$ are respectively replaced by $\alpha$ and $c$, we know that there exists two matrices $S_{n_{1}}$ and $S_{n_{2}}$ which only depend on $\alpha$ and $c$ such that:

$$
\forall t \geq 0, A_{n_{i}}^{T}(t) S_{n_{i}}+S_{n_{i}} A_{n_{i}}(t)-\rho_{i} C_{n_{i}}^{T} C_{n_{i}} \leq-\eta_{i} I_{n_{i}}
$$

for some constants $\rho_{i}>0$ and $\eta_{i}>0, i=1,2$.

In what follows, we will show that system (9) in which the $S_{n_{i}}$ 's are those satisfying (17), is an exponential estimator.

Set $e(t)=\hat{x}(t)-x(t)=\left[\begin{array}{l}e^{1}(t) \\ e^{2}(t)\end{array}\right]=\left[\begin{array}{l}\hat{x}^{1}(t)-x^{1}(t) \\ \hat{x}^{2}(t)-x^{2}(t)\end{array}\right]$, where $x(t)$ and $\hat{x}(t)$ are two respective trajectories of systems $(8)$.

Differentiating $e(t)$, we obtain:

$$
\left\{\begin{array}{l}
\dot{e}(t)^{1}(t)=f^{1}(\hat{x}(t), u(t))-f^{1}(x(t), u(t)) \\
-r_{1} \Delta_{\theta^{\delta_{1}}} S_{n_{1}}^{-1} C_{n_{1}}^{T}\left(C_{n_{1}} \hat{x}^{1}(t)-y_{1}(t)\right)+d^{1}(t) \\
\dot{e}^{2}(t)=f^{2}(\hat{x}(t), u(t))-f^{2}(x(t), u(t)) \\
-r_{2} \Delta_{\theta^{\delta_{2}}} S_{n_{2}}^{-1} C_{n_{2}}^{T}\left(C_{n_{2}} \hat{x}^{2}(t)-y_{2}(t)\right)+d^{2}(t)
\end{array}\right.
$$

Recall that:

$$
\left\{\begin{array}{l}
f_{j}^{1}=f_{j}^{1}\left(x_{1}^{1}(t), \ldots, x_{j+1}^{1}(t), u(t)\right) ; j=1, \ldots, n_{1}-1 \\
f_{n_{1}}^{1}=f_{n_{1}}^{1}(x, u) \\
f_{j}^{2}=f_{j}^{2}\left(x_{1}^{2}, \ldots, x_{j+1}^{2}, u\right) ; j=1, \ldots, n_{2}-2 \\
f_{n_{2}-1}^{2}=f_{n_{2}-1}^{2}(x, u) \\
f_{n_{2}}^{2}=f_{n_{2}}^{2}(x, u)
\end{array}\right.
$$


Using the following notations:

$$
\left\{\begin{array}{l}
\delta f_{j}^{1}=f_{j}^{1}\left(\hat{x}_{1}^{1}, \ldots, \hat{x}_{j}^{1}, x_{j+1}^{1}, u\right)-f_{j}^{1}\left(x_{1}^{1}, \ldots, x_{j+1}^{1}, u\right) ; j=1, \ldots, n_{1}-1 \\
\delta f_{n_{1}}^{1}=f_{n_{1}}^{1}(\hat{x}, u)-f_{n_{1}}^{1}(x, u) \\
\delta f_{j}^{2}=f_{j}^{2}\left(\hat{x}_{1}^{2}, \ldots, \hat{x}_{j}^{2}, x_{j+1}^{2}, u\right)-f_{j}^{2}\left(x_{1}^{2}, \ldots, x_{j+1}^{2}, u\right) ; j=1, \ldots, n_{2}-2 \\
\delta f_{n_{2}-1}^{2}=f_{n_{2}-1}^{2}\left(\hat{x}^{1}, \hat{x}_{1}^{2}, \ldots, \hat{x}_{n_{2}-1}^{2}, x_{n_{2}}^{2}, u\right)-f_{n_{2}-1}^{2}\left(x^{1}, x_{1}^{2}, \ldots, x_{n_{2}}^{2}, u\right) \\
\delta f_{n_{2}}^{2}=f_{n_{2}}^{2}(\hat{x}, u)-f_{n_{2}}^{2}(x, u)
\end{array}\right.
$$

we obtain:

for $1 \leq j \leq n_{1}-1$;

$$
\begin{aligned}
f_{j}^{1}(\hat{x}, u)-f_{j}^{1}(x, u) & =f_{j}^{1}\left(\hat{x}_{1}^{1}, \ldots, \hat{x}_{j+1}^{1}, u\right)-f_{j}^{1}\left(\hat{x}_{1}^{1}, \ldots, \hat{x}_{j}^{1}, x_{j+1}^{1}, u\right)+\delta f_{j}^{1} \\
& =\frac{\partial f_{j}^{1}}{\partial x_{j+1}^{1}}\left(\hat{x}_{1}^{1}, \ldots, \hat{x}_{j}^{1}, \sigma_{j}^{1}(t), u(t)\right) e_{j+1}^{1}(t)+\delta f_{j}^{1}
\end{aligned}
$$

where $\sigma_{j}^{1}(t)=\hat{x}_{j+1}^{1}(t)+\tau_{j}(t) e_{j+1}^{1}(t)$ for some $\tau_{j}(t), 0 \leq \tau_{j}(t) \leq 1$.

Similarly, we have:

$$
f_{j}^{2}(\hat{x}, u)-f_{j}^{2}(x, u)=\frac{\partial f_{j}^{2}}{\partial x_{j+1}^{2}}\left(\hat{x}_{1}^{2}, \ldots, \hat{x}_{j}^{2}, \sigma_{j}^{2}(t), u(t)\right) e_{j+1}^{2}(t)+\delta f_{j}^{2} ; \text { for } 1 \leq j \leq n_{2}-2
$$

and,

$$
f_{n_{2}-1}^{2}(\hat{x}, u)-f_{n_{2}-1}^{2}(x, u)=\frac{\partial f_{n_{2}-1}^{2}}{\partial x_{n_{2}}^{2}}\left(\hat{x}^{1}, \hat{x}_{1}^{2}, \ldots, \hat{x}_{n_{2}-1}^{2}, \sigma_{j}^{2}(t), u(t)\right) e_{n_{2}}^{2}(t)+\delta f_{n_{2}-1}^{2}
$$

where $\sigma_{j}^{2}(t)=\hat{x}_{j+1}^{2}(t)+\tau_{j}^{\prime}(t) e_{j+1}^{2}(t)$ for some $\tau_{j}^{\prime}(t), 0 \leq \tau_{j}^{\prime}(t) \leq 1$.

Now set:

$$
\begin{aligned}
& a_{j}^{1}(t)=\frac{\partial f_{j}^{1}}{\partial x_{j+1}^{1}}\left(\hat{x}_{1}^{1}(t), \ldots, \hat{x}_{j}^{1}(t), \sigma_{j}^{1}(t)\right) ; \text { for } j=1, \ldots, n_{1}-1 \\
& a_{j}^{2}(t)=\frac{\partial f_{j}^{2}}{\partial x_{j+1}^{2}}\left(\hat{x}_{1}^{2}(t), \ldots, \hat{x}_{j}^{2}(t), \sigma_{j}^{2}(t)\right) ; \text { for } j=1, \ldots, n_{2}-2 \\
& a_{n_{2}-1}^{1}(t)=\frac{\partial f_{j}^{2}}{\partial x_{n_{2}}^{2}}\left(\hat{x}^{1}, \hat{x}_{1}^{2}, \ldots, \hat{x}_{n_{2}-1}^{2}, \sigma_{n_{2}}^{2}(t), u(t)\right) \\
& A_{n_{i}}(t)=\left[\begin{array}{ccccc}
0 & a_{1}^{i}(t) & 0 & \ldots & 0 \\
\vdots & & a_{2}^{i}(t) & & \vdots \\
0 & & & \ddots & a_{n_{i}-1}^{i}(t) \\
0 & \ldots & & 0 & 0
\end{array}\right]
\end{aligned}
$$

The error equation (18) becomes:

$$
\left\{\begin{array}{l}
\dot{e}^{1}(t)=A_{n_{1}}(t) e^{1}(t)+\delta f^{1}-r_{1} \Delta_{\theta^{\delta_{1}}} S_{n_{1}}^{-1} C_{n_{1}}^{T} C_{n_{1}} e^{1}(t)+d^{1}(t) \\
\dot{e}^{2}(t)=A_{n_{2}}(t) e^{2}(t)+\delta f^{2}-r_{2} \Delta_{\theta^{\delta}} S_{n_{2}}^{-1} C_{n_{2}}^{T} C_{n_{2}} e^{2}(t)+d^{2}(t)
\end{array}\right.
$$


where $\delta f^{1}=\left[\delta f_{1}^{1}, \ldots, \delta f_{n_{1}}^{1}\right]^{T}$ and $\delta f^{2}=\left[\delta f_{1}^{2}, \ldots, \delta f_{n_{2}}^{2}\right]^{T}$.

As in ${ }^{5}$, we use the following change of coordinates:

$$
\left\{\begin{array}{l}
\varepsilon_{j}^{1}=\theta^{-j \delta_{1}} e_{j}^{1} \text { for } j=1, \ldots, n_{1} \\
\varepsilon_{j}^{2}=\theta^{-j \delta_{2}} e_{j}^{2} \text { for } j=1, \ldots, n_{2}
\end{array}\right.
$$

Set $\varepsilon^{1}=\left[\varepsilon_{1}^{1}, \ldots, \varepsilon_{n_{1}}^{1}\right]^{T}, \varepsilon^{2}=\left[\varepsilon_{1}^{2}, \ldots, \varepsilon_{n_{2}}^{2}\right]^{T}$ and $\varepsilon=\left[\begin{array}{c}\varepsilon^{1} \\ \varepsilon^{2}\end{array}\right]$, this change of coordinates takes the vectorial form:

$$
\varepsilon=\left[\begin{array}{l}
\varepsilon^{1} \\
\varepsilon^{2}
\end{array}\right]=\left[\begin{array}{c}
\Delta_{\theta^{\delta_{1}}}^{-1} e^{1} \\
\Delta_{\theta^{\delta_{2}}}^{-1} e^{2}
\end{array}\right]
$$

A simple calculation shows that the error equation (20) is equivalent to:

$$
\left\{\begin{array}{l}
\dot{\varepsilon}^{1}(t)=\theta^{\delta_{1}}\left(A_{n_{1}}(t)-r_{1} S_{n_{1}}^{-1} C_{n_{1}}^{T} C_{n_{1}}\right) \varepsilon^{1}(t)+\Delta_{\theta^{\delta_{1}}}^{-1}\left(\delta f^{1}+d^{1}(t)\right) \\
\dot{\varepsilon}^{2}(t)=\theta^{\delta_{2}}\left(A_{n_{2}}(t)-r_{2} S_{n_{2}}^{-1} C_{n_{2}}^{T} C_{n_{2}}\right) \varepsilon^{2}(t)+\Delta_{\theta^{\delta_{2}}}^{-1}\left(\delta f^{2}+d^{2}(t)\right)
\end{array}\right.
$$

To end the proof of theorem 2 , we only need to show that for $\theta$ sufficiently large, we have:

$$
\|\varepsilon(t)\| \leq c_{1} e^{-\sigma t}+c_{2} d
$$

for some positive constants $c_{1}, c_{2}$ and $\sigma$.

To do so, we will show that the quadratic form $V(\varepsilon)=\left(\varepsilon^{1}\right)^{T} S_{n_{1}} \varepsilon^{1}+\left(\varepsilon^{2}\right)^{T} S_{n_{2}} \varepsilon^{2}$ is a Lyapunov function for system (21). Let $\varepsilon(t)$ be a trajectory of (21), and differentiate $V(\varepsilon(t))$, we get:

$$
\begin{aligned}
\dot{V}(\varepsilon(t)) & =\theta^{\delta_{1}}\left(\varepsilon^{1}(t)\right)^{T}\left[A_{n_{1}}(t)^{T} S_{n_{1}}+S_{n_{1}} A_{n_{1}}(t)-2 r_{1} C_{n_{1}}^{T} C_{n_{1}}\right] \varepsilon^{1}(t) \\
& +\theta^{\delta_{2}}\left(\varepsilon^{2}(t)\right)^{T}\left[A_{n_{2}}(t)^{T} S_{n_{2}}+S_{n_{2}} A_{n_{2}}(t)-2 r_{2} C_{n_{2}}^{T} C_{n_{2}}\right] \varepsilon^{2}(t) \\
& +2\left(\varepsilon^{1}(t)\right)^{T} S_{n_{1}} \Delta_{\theta^{\delta}}^{-1}\left(\delta f^{1}+d^{1}(t)\right)+2\left(\varepsilon^{2}(t)\right)^{T} S_{n_{2}} \Delta_{\theta^{\delta_{2}}}^{-1}\left(\delta f^{2}+d^{2}(t)\right)
\end{aligned}
$$

Now, choose $r_{i}=\frac{\rho_{i}}{2}$ and using inequality (17), we get:

$$
\begin{aligned}
\dot{V}(\varepsilon(t)) & \leq-\theta^{\delta_{1}} \eta_{1}\left\|\varepsilon^{1}(t)\right\|^{2}-\theta^{\delta_{2}} \eta_{2}\left\|\varepsilon^{2}(t)\right\|^{2} \\
& +2\left(\varepsilon^{1}(t)\right)^{T} S_{n_{1}} \Delta_{\theta^{\delta_{1}}}^{-1} \delta f^{1}+2\left(\varepsilon^{2}(t)\right)^{T} S_{n_{2}} \Delta_{\theta^{\delta_{2}}}^{-1} \delta f^{2} \\
& +2\left(\varepsilon^{1}(t)\right)^{T} S_{n_{1}} \Delta_{\theta^{\delta_{1}}}^{-1} d^{1}(t)+2\left(\varepsilon^{2}(t)\right)^{T} S_{n_{2}} \Delta_{\theta^{\delta_{2}}}^{-1} d^{2}(t) \\
& \leq-\theta^{\delta_{1}} \eta_{1}\left\|\varepsilon^{1}(t)\right\|^{2}-\theta^{\delta_{2}} \eta_{2}\left\|\varepsilon^{2}(t)\right\|^{2} \\
& +2 \sqrt{\left(\varepsilon^{1}(t)\right)^{T} S_{n_{1}} \varepsilon^{1}(t)} \sqrt{\left(\Delta_{\theta^{\delta_{1}}}^{-1} \delta f^{1}\right)^{T} S_{n_{1}} \Delta_{\theta^{\delta_{1}}}^{-1} \delta f^{1}} \\
& +2 \sqrt{\left(\varepsilon^{2}(t)\right)^{T} S_{n_{2}} \varepsilon^{2}(t)} \sqrt{\left(\Delta_{\theta^{\delta_{2}}}^{-1} \delta f^{2}\right)^{T} S_{n_{2}} \Delta_{\theta^{\delta_{2}}}^{-1} \delta f^{2}} \\
& +2 \sqrt{\left(\varepsilon^{1}(t)\right)^{T} S_{n_{1}} \varepsilon^{1}(t)} \sqrt{\left(\Delta_{\theta^{\delta_{1}}}^{-1} d^{1}(t)\right)^{T} S_{n_{1}} \Delta_{\theta^{\delta_{1}}}^{-1} d^{1}(t)} \\
& +2 \sqrt{\left(\varepsilon^{2}(t)\right)^{T} S_{n_{2}} \varepsilon^{2}(t)} \sqrt{\left(\Delta_{\theta^{\delta_{2}}}^{-1} d^{2}(t)\right)^{T} S_{n_{2}} \Delta_{\theta^{\delta_{2}}}^{-1} d^{2}(t)}
\end{aligned}
$$

Recall that the following inequalities hold for any S.P.D matrix $S$ :

$$
\lambda_{\min }(S)\|X\|^{2} \leq X^{T} S X \leq \lambda_{\max }(S)\|X\|^{2}
$$


where $\lambda_{\min }(S)$ and $\lambda_{\max }(S)$ are respectively the smallest and the largest eigenvalues of $S$. Applying these inequalities, we obtain:

$$
\begin{aligned}
\dot{V}(\varepsilon(t)) & \leq-\frac{\theta^{\delta_{1}} \eta_{1}}{\lambda_{\max }\left(S_{n_{1}}\right)}\left(\varepsilon^{1}(t)\right)^{T} S_{n_{1}} \varepsilon^{1}(t)-\frac{\theta^{\delta_{2}} \eta_{2}}{\lambda_{\max }\left(S_{n_{2}}\right)}\left(\varepsilon^{2}(t)\right)^{T} S_{n_{2}} \varepsilon^{2}(t) \\
& +2 \sqrt{\lambda_{\max }\left(S_{n_{1}}\right)} \sqrt{\left(\varepsilon^{1}(t)\right)^{T} S_{n_{1}} \varepsilon^{1}(t)}\left\|\Delta_{\theta^{\delta_{1}}}^{-1} \delta f^{1}\right\| \\
& +2 \sqrt{\lambda_{\max }\left(S_{n_{2}}\right)} \sqrt{\left(\varepsilon^{2}(t)\right)^{T} S_{n_{2}} \varepsilon^{2}(t)}\left\|\Delta_{\theta^{\delta_{2}}}^{-1} \delta f^{2}\right\| \\
& +2 \sqrt{\lambda_{\max }\left(S_{n_{1}}\right)} \sqrt{\left(\varepsilon^{1}(t)\right)^{T} S_{n_{1}} \varepsilon^{1}(t)}\left\|\Delta_{\theta^{\delta_{1}}}^{-1} d^{1}(t)\right\| \\
& +2 \sqrt{\lambda_{\max }\left(S_{n_{2}}\right)} \sqrt{\left(\varepsilon^{2}(t)\right)^{T} S_{n_{2}} \varepsilon^{2}(t)}\left\|\Delta_{\theta^{\delta_{2}}}^{-1} d^{2}(t)\right\|
\end{aligned}
$$

Recall that $\Delta_{\theta^{\delta_{i}}}^{-1} \delta f^{i}=\left[\begin{array}{c}\frac{1}{\theta^{\delta_{i}}} \delta f_{1}^{i} \\ \vdots \\ \frac{1}{\theta^{n_{i} \delta_{i}}} \delta f_{n_{i}}^{i}\end{array}\right]$, and using $\left.H_{3}\right)-i$ ) and the triangular structure of the $\left(\delta f_{j}^{i}\right)$ 's we obtain:

For $j=1, \ldots, n_{1}-1 ; \theta^{-j \delta_{1}}\left|\delta f_{j}^{1}\right| \leq c \theta^{-j \delta_{1}} \sqrt{\left(e_{1}^{1}\right)^{2}+\ldots,\left(e_{j}^{1}\right)^{2}}$ where $c$ is the Lipschitz constant of the $f^{i}$ s.

Hence,

$$
\theta^{-j \delta_{1}}\left|\delta f_{j}^{1}\right| \leq c \theta^{-j \delta_{1}} \sqrt{\left(\theta^{\delta_{1}} \varepsilon_{1}^{1}(t)\right)^{2}+\ldots,\left(\theta^{j \delta_{1}} \varepsilon_{j}^{1}(t)\right)^{2}}
$$

Now, taking $\theta \geq 1$, we get:

$$
\theta^{-j \delta_{1}}\left|\delta f_{j}^{1}\right| \leq c \sqrt{\left(\varepsilon_{1}^{1}(t)\right)^{2}+\ldots,\left(\varepsilon_{j}^{1}(t)\right)^{2}} \leq c\left\|\varepsilon^{1}(t)\right\|
$$

For $j=n_{1}$, we have:

$$
\begin{aligned}
\theta^{-n_{1} \delta_{1}}\left|\delta f_{n_{1}}^{1}\right| & \leq c \theta^{-n_{1} \delta_{1}}\|e(t)\| \\
& =c \theta^{-n_{1} \delta_{1}} \sqrt{\left(\theta^{\delta_{1}} \varepsilon_{1}^{1}(t)\right)^{2}+\ldots+\left(\theta^{n_{1} \delta_{1}} \varepsilon_{n_{1}}^{1}(t)\right)^{2}+\left(\theta^{\delta_{2}} \varepsilon_{1}^{2}(t)\right)^{2}+\ldots+\left(\theta^{n_{2} \delta_{2}} \varepsilon_{n_{2}}^{2}(t)\right)^{2}}
\end{aligned}
$$

For $\theta \geq 1$, we obtain:

$$
\theta^{-n_{1} \delta_{1}}\left|\delta f_{n_{1}}^{1}\right| \leq c\left\|\varepsilon^{1}(t)\right\|+c \theta^{n_{2} \delta_{2}-n_{1} \delta_{1}}\left\|\varepsilon^{2}(t)\right\|
$$

Similarly for $\theta \geq 1$, we have:

$$
\theta^{-j \delta_{2}}\left|\delta f_{j}^{2}\right| \leq c\left\|\varepsilon^{2}(t)\right\| \text { for } 1 \leq j \leq n_{2}
$$

For $j=n_{2}-1, n_{2}$, and $\theta \geq 1$, we obtain:

$$
\left\{\begin{array}{c}
\theta^{-\left(n_{2}-1\right) \delta_{2}}\left|\delta f_{n_{2}-1}^{2}\right| \leq c\left\|\varepsilon^{2}(t)\right\|+c \theta^{n_{1} \delta_{1}-\left(n_{2}-1\right) \delta_{2}}\left\|\varepsilon^{1}(t)\right\|,\left(j=n_{2}-1\right) \\
\theta^{-n_{2} \delta_{2}}\left|\delta f_{n_{2}}^{2}\right| \leq c\left\|\varepsilon^{2}(t)\right\|+c \theta^{n_{1} \delta_{1}-n_{2} \delta_{2}}\left\|\varepsilon^{1}(t)\right\|,\left(j=n_{2}\right) \\
\leq c\left\|\varepsilon^{2}(t)\right\|+c \theta^{n_{1} \delta_{1}-\left(n_{2}-1\right) \delta_{2}}\left\|\varepsilon^{1}(t)\right\|(\text { since } \theta \geq 1)
\end{array}\right.
$$

Finally, inequalities (24) to (27) with $\theta \geq 1$, lead to:

$$
\left\{\begin{array}{c}
\left\|\Delta_{\theta^{\delta_{1}}}^{-1} \delta f^{1}\right\| \leq c\left\|\varepsilon^{1}(t)\right\|+c \theta^{n_{2} \delta_{2}-n_{1} \delta_{1}}\left\|\varepsilon^{2}(t)\right\| \\
\left\|\Delta_{\theta^{\delta_{2}}} \delta f^{2}\right\| \leq c \theta^{n_{1} \delta_{1}-\left(n_{2}-1\right) \delta_{2}}\left\|\varepsilon^{1}(t)\right\|+c\left\|\varepsilon^{2}(t)\right\|
\end{array}\right.
$$


hence;

$$
\left\{\begin{array}{c}
\left\|\Delta_{\theta^{\delta_{1}}}^{-1} \delta f^{1}\right\| \leq \frac{c}{\sqrt{\lambda_{\min }\left(S_{n_{1}}\right)}} \sqrt{\varepsilon^{1}(t)^{T} S_{n_{1}} \varepsilon^{1}(t)}+\frac{c \theta^{n_{2} \delta_{2}-n_{1} \delta_{1}}}{\sqrt{\lambda_{\min }\left(S_{n_{2}}\right)}} \sqrt{\varepsilon^{2}(t)^{T} S_{n_{2}} \varepsilon^{2}(t)} \\
\left\|\Delta_{\theta^{\delta_{2}}} \delta f^{2}\right\| \leq \frac{c \theta^{n_{1} \delta_{1}-\left(n_{2}-1\right) \delta_{2}}}{\sqrt{\lambda_{\min }\left(S_{n_{1}}\right)}} \sqrt{\varepsilon^{1}(t)^{T} S_{n_{1}} \varepsilon^{1}(t)}+\frac{c}{\sqrt{\lambda_{\min }\left(S_{n_{2}}\right)}} \sqrt{\varepsilon^{2}(t)^{T} S_{n_{2}} \varepsilon^{2}(t)}
\end{array}\right.
$$

Combining (23) and (28), we obtain:

$$
\begin{aligned}
& \dot{V}(\varepsilon(t)) \leq-\left(\frac{\theta^{\delta_{1}} \eta_{1}}{\lambda_{\max }\left(S_{n_{1}}\right)}-2 c \frac{\sqrt{\lambda_{\max }\left(S_{n_{1}}\right)}}{\sqrt{\lambda_{\min }\left(S_{n_{1}}\right)}}\right)\left(\varepsilon^{1}(t)\right)^{T} S_{n_{1}} \varepsilon^{1}(t) \\
& \quad-\left(\frac{\theta^{\delta_{2}} \eta_{2}}{\lambda_{\max }\left(S_{n_{2}}\right)}-2 c \frac{\sqrt{\lambda_{\max }\left(S_{n_{2}}\right)}}{\sqrt{\lambda_{\min }\left(S_{n_{2}}\right)}}\right)\left(\varepsilon^{2}(t)\right)^{T} S_{n_{2}} \varepsilon^{2}(t) \\
& +2 c\left(\theta^{n_{2} \delta_{2}-n_{1} \delta_{1}} \frac{\sqrt{\lambda_{\max }\left(S_{n_{1}}\right)}}{\sqrt{\lambda_{\min }\left(S_{n_{2}}\right)}}+\theta^{n_{1} \delta_{1}-\left(n_{2}-1\right)} \delta_{2} \frac{\sqrt{\lambda_{\max }\left(S_{n_{2}}\right)}}{\sqrt{\lambda_{\min }\left(S_{n_{1}}\right)}}\right) \sqrt{\left(\varepsilon^{1}(t)\right)^{T} S_{n_{1}} \varepsilon^{1}(t)} \sqrt{\varepsilon^{2}(t)^{T} S_{n_{1}} \varepsilon^{2}(t)} \\
& +2 \sqrt{\lambda_{\max }\left(S_{n_{1}}\right)} \sqrt{\left(\varepsilon^{1}(t)\right)^{T} S_{n_{2}} \varepsilon^{1}(t)}\left\|\Delta_{\theta^{\delta_{1}}}^{-1} d^{1}(t)\right\| \\
& +2 \sqrt{\lambda_{\max }\left(S_{n_{2}}\right)} \sqrt{\left(\varepsilon^{2}(t)\right)^{T} S_{n_{2}} \varepsilon^{2}(t) \| \Delta_{\theta^{\delta_{2}}}^{-1}} d^{2}(t) \|
\end{aligned}
$$

Setting $W_{1}=\theta^{\frac{\delta_{1}}{2}} \sqrt{\left(\varepsilon^{1}(t)\right)^{T} S_{n_{1}} \varepsilon^{1}(t)}, W_{2}=\theta^{\frac{\delta_{2}}{2}} \sqrt{\left(\varepsilon^{2}(t)\right)^{T} S_{n_{2}} \varepsilon^{2}(t)}$, we obtain:

$$
\begin{aligned}
\dot{V}(\varepsilon(t)) & \leq-\left(\frac{\eta_{1}}{\lambda_{\max }\left(S_{n_{1}}\right)}-2 c \frac{\theta^{-\delta_{1}} \sqrt{\lambda_{\max }\left(S_{n_{1}}\right)}}{\sqrt{\lambda_{\min }\left(S_{n_{1}}\right)}}\right) W_{1} \\
& -\left(\frac{\eta_{2}}{\lambda_{\max }\left(S_{n_{2}}\right)}-2 c \frac{\theta^{-\delta_{2}} \sqrt{\lambda_{\max }\left(S_{n_{2}}\right)}}{\sqrt{\lambda_{\min }\left(S_{n_{2}}\right)}}\right) W_{2} \\
& +2 c\left(\theta^{\left(n_{2} \delta_{2}-n_{1} \delta_{1}-\frac{\delta_{1}}{2}-\frac{\delta_{2}}{2}\right)} \frac{\sqrt{\lambda_{\max }\left(S_{n_{1}}\right)}}{\sqrt{\lambda_{\min }\left(S_{n_{2}}\right)}}\right. \\
& \left.+\theta^{\left(n_{1} \delta_{1}-\left(n_{2}-1\right) \delta_{2}-\frac{\delta_{1}}{2}-\frac{\delta_{2}}{2}\right)} \frac{\sqrt{\lambda_{\max }\left(S_{n_{2}}\right)}}{\sqrt{\lambda_{\min }\left(S_{n_{1}}\right)}}\right) \sqrt{W_{1}} \sqrt{W_{2}} \\
& +2 \theta^{-\frac{\delta_{1}}{2}} \sqrt{\lambda_{\max }\left(S_{n_{1}}\right)} \sqrt{W_{1}\left\|\Delta_{\theta^{\delta_{1}}}^{-1} d^{1}(t)\right\|}+2 \theta^{-\frac{\delta_{2}}{2}} \sqrt{\lambda_{\max }\left(S_{n_{2}}\right)} \sqrt{W_{2}}\left\|\Delta_{\theta^{\delta_{2}}}^{-1} d^{2}(t)\right\|
\end{aligned}
$$

Set $\left.\alpha(\theta)=c\left(\theta^{\left(n_{2} \delta_{2}-n_{1} \delta_{1}-\frac{\delta_{1}}{2}-\frac{\delta_{2}}{2}\right.}\right) \frac{\sqrt{\lambda_{\max }\left(S_{n_{1}}\right)}}{\sqrt{\lambda_{\min }\left(S_{n_{2}}\right)}}+\theta^{\left(n_{1} \delta_{1}-\left(n_{2}-1\right) \delta_{2}-\frac{\delta_{1}}{2}-\frac{\delta_{2}}{2}\right)} \frac{\sqrt{\lambda_{\max }\left(S_{n_{2}}\right)}}{\sqrt{\lambda_{\min }\left(S_{n_{1}}\right)}}\right)$, we get:

$$
\begin{aligned}
\dot{V}(\varepsilon(t)) & \leq-\left(\frac{\eta_{1}}{\lambda_{\max }\left(S_{n_{1}}\right)}-2 c \frac{\theta^{-\delta_{1}} \sqrt{\lambda_{\max }\left(S_{n_{1}}\right)}}{\sqrt{\lambda_{\min }\left(S_{n_{1}}\right)}}-\alpha(\theta)\right) W_{1} \\
& -\left(\frac{\eta_{2}}{\lambda_{\max }\left(S_{n_{2}}\right)}-2 c \frac{\theta^{-\delta_{2}} \sqrt{\lambda_{\max }\left(S_{n_{2}}\right)}}{\sqrt{\lambda_{\min }\left(S_{n_{2}}\right)}}-\alpha(\theta)\right) W_{2} \\
& +2 \theta^{-\frac{\delta_{1}}{2}} \sqrt{\lambda_{\max }\left(S_{n_{1}}\right)} \sqrt{W_{1}}\left\|\Delta_{\theta^{\delta_{1}}}^{-1} d^{1}(t)\right\| \\
& +2 \theta^{-\frac{\delta_{2}}{2}} \sqrt{\lambda_{\max }\left(S_{n_{2}}\right)} \sqrt{W_{2}}\left\|\Delta_{\theta^{\delta_{2}}}^{-1} d^{2}(t)\right\|
\end{aligned}
$$

Now using the condition of theorem 2: $\frac{2 n_{1}-1}{2 n_{2}-1} \delta_{1}<\delta_{2}<\frac{2 n_{1}+1}{2 n_{2}-1} \delta_{1}$, it follows that:

$$
n_{1} \delta_{1}-\left(n_{2}-1\right) \delta_{2}-\frac{\delta_{1}}{2}-\frac{\delta_{2}}{2}<0
$$

Hence there exist $\theta_{0} \geq 1$ such that for every $\theta \geq \theta_{0}$, we have:

$$
\left\{\begin{aligned}
2 c \frac{\theta^{-\delta_{1}} \sqrt{\lambda_{\max }\left(S_{n_{1}}\right)}}{\sqrt{\lambda_{\min }\left(S_{n_{1}}\right)}}+\alpha(\theta) & \leq \frac{\eta_{1}}{2 \lambda_{\max }\left(S_{n_{1}}\right)} \\
2 c \frac{\theta^{-\delta_{2}} \sqrt{\lambda_{\max }\left(S_{n_{2}}\right)}}{\sqrt{\lambda_{\min }\left(S_{n_{2}}\right)}}+\alpha(\theta) & \leq \frac{\eta_{2}}{2 \lambda_{\max }\left(S_{n_{2}}\right)}
\end{aligned}\right.
$$


Combining (29) and (30), and taking $\theta \geq \theta_{0}$, we deduce that:

$$
\begin{aligned}
\dot{V}(\varepsilon(t)) & \leq-\frac{\eta_{1}}{2 \lambda_{\max }\left(S_{n_{1}}\right)} W_{1}-\frac{\eta_{2}}{2 \lambda_{\max }\left(S_{n_{2}}\right)} W_{2} \\
& +2 \theta^{-\frac{3 \delta_{1}}{2}} \sqrt{\lambda_{\max }\left(S_{n_{1}}\right)} d^{1} \sqrt{W_{1}}+2 \theta^{-\frac{3 \delta_{2}}{2}} \sqrt{\lambda_{\max }\left(S_{n_{2}}\right)} d^{2} \sqrt{W_{2}}
\end{aligned}
$$

where $d=\sup _{t \geq 0}\left\{\left\|d_{i}(t)\right\| ; i=1,2\right\}$

Hence,

$$
\begin{aligned}
\dot{V}(\varepsilon(t)) & \leq-\frac{\eta_{1} \theta^{\delta_{1}}}{2 \lambda_{\max }\left(S_{n_{1}}\right)}\left(\varepsilon^{1}(t)\right)^{T} S_{n_{1}} \varepsilon^{1}(t)-\frac{\eta_{2} \theta^{\delta_{2}}}{2 \lambda_{\max }\left(S_{n_{2}}\right)}\left(\varepsilon^{2}(t)\right)^{T} S_{n_{2}} \varepsilon^{2}(t) \\
& +2 d \theta^{-\delta_{1}} \sqrt{\lambda_{\max }\left(S_{n_{1}}\right)} \sqrt{\left(\varepsilon^{1}(t)\right)^{T} S_{n_{1}} \varepsilon^{1}(t)} \\
& +2 d \theta^{-\delta_{2}} \sqrt{\lambda_{\max }\left(S_{n_{2}}\right)} \sqrt{\left(\varepsilon^{2}(t)\right)^{T} S_{n_{2}} \varepsilon^{2}(t)} \\
& \leq-\sigma(\theta) V(\varepsilon(t))+\gamma(\theta) d \sqrt{2} \sqrt{V(\varepsilon(t))}
\end{aligned}
$$

where

$\sigma(\theta)=\min \left\{\frac{\eta_{1} \theta^{\delta_{1}}}{2 \lambda_{\max }\left(S_{n_{1}}\right)}, \frac{\eta_{2} \theta^{\delta_{2}}}{2 \lambda_{\max }\left(S_{n_{2}}\right)}\right\} ; \gamma(\theta)=\max \left\{2 \theta^{-\delta_{1}} \sqrt{\lambda_{\max }\left(S_{n_{1}}\right)}, 2 \theta^{-\delta_{2}} \sqrt{\lambda_{\max }\left(S_{n_{2}}\right)}\right\}$ and, $d=\max \left\{d^{1}, d^{2}\right\}$.

Hence the ball $B\left(0, \frac{\gamma(\theta) d}{\sigma(\theta)}\right)$ exponentially attracts $\varepsilon(t)$.

Since $e(t)=\hat{x}(t)-x(t)=\left[\left(\Delta_{\theta^{\delta_{1}}} \varepsilon^{1}(t)\right)^{T} \Delta_{\theta^{\delta_{2}}} \varepsilon^{2}(t)\right]^{T}$ it follows that $e(t)$ is exponentially attracted by some $B\left(0, \lambda_{1}(\theta) d\right)$.

\section{APPLICATION TO BINARY DISTLLATION COLUMNS}

Based on the Lewis assumption (the molar overflow is constant), the model of a binary distillation column that we consider is the classical $(L, V)$ model:

$$
\left\{\begin{array}{l}
H_{1} \dot{x}_{1}=V\left(y_{2}-x_{1}\right)(\text { total condenser }) \\
H_{i} \dot{x}_{i}=L\left(x_{i-1}-x_{i}\right)+V\left(y_{i+1}-y_{i}\right)(i=2, \ldots, f-1 ; \text { rectifying section }) \\
H_{f} \dot{x}_{f}=F\left(Z_{f}-x_{f}\right)+L\left(x_{f-1}-x_{f}\right)+V\left(y_{f+1}-y_{f}\right)(\text { feed tray) } \\
H_{i} \dot{x}_{i}=(F+L)\left(x_{i-1}-x_{i}\right)+V\left(y_{i+1}-y_{i}\right)(i=f+1, \ldots, n-1 ; \text { stripping section }) \\
H_{n} \dot{x}_{n}=(F+L)\left(x_{n-1}-x_{n}\right)+V\left(x_{n}-y_{n}\right)(\text { boiler })
\end{array}\right.
$$

Where $H_{i}$ is the liquid holdup on the ith-tray supposed known, $x_{i}, y_{i}$ are the liquid and vapor compositions on the $i$ th-tray, $f$ is the number of the feed tray, $F, L, V$ are the feed, reflux, and vapor rates (measured), and $Z F$ is the feed composition. On each tray the liquid and vapor compositions, $y_{i}$ and $x_{i}$, are linked by the liquid-vapor equilibrium law:

$$
y_{i}=\frac{\alpha x_{i}}{1+(\alpha-1) x_{i}}
$$

where $\alpha$ is the relative volatility constant $(0<\alpha<1)$. The state of the model are the set of liquid and feed compositions $\left(x_{i}, Z_{f}\right)$ of the most volatile component. The two control variables are $L$ and $V$, i.e. $u=[L, V]^{T}$. In practice, the top and bottom product compositions $x_{1}$ and $x_{n}$ are measured, i.e. $y=\left[x_{1}, x_{n}\right]^{T}$. In the sequel, we will use the following notations: 


$$
\left\{x^{1}=\left[\begin{array}{c}
x_{1}^{1} \\
\cdot \\
\cdot \\
x_{n_{1}}^{1}
\end{array}\right]=\left[\begin{array}{c}
x_{1} \\
\cdot \\
\cdot \\
x_{f-1}
\end{array}\right] ; x^{2}=\left[\begin{array}{c}
x_{1}^{2} \\
\cdot \\
\cdot \\
x_{n_{2}}^{2}
\end{array}\right]=\left[\begin{array}{c}
x_{n} \\
\cdot \\
\cdot \\
x_{f} \\
Z_{f}
\end{array}\right]\right.
$$

Here, $n_{1}=f-1, n_{2}=n-f+2$, The dynamics of $Z_{f}$ is assumed to be unknown and bounded:

$$
\dot{Z}_{f}=\varepsilon(t)
$$

The extended model is then of the form (9):

$$
\left\{\begin{array}{l}
\dot{x}^{1}(t)=f^{1}(x(t), u(t)) \\
\dot{x}^{2}(t)=f^{2}(x(t), u(t))+d^{2}(t) \\
y(t)=\left(x_{1}^{1}, x_{1}^{2}\right)^{T}=\left(x_{1}, x_{n}\right)^{T}
\end{array}\right.
$$

where $d_{n_{2}}^{2}(t)=\varepsilon(t)$ and $d_{i}^{2}(t)=0$, for $1 \leq i \leq n_{2}-1$.

In order to apply the observer design of section 2, one has to check hypothesis $H_{3}$ ). Let us check $H_{3}$ ):

$\left.H_{3}\right)$-i) can be obtained by extending nonlinear dynamics by global Lipschitz one. Indeed, the state components of the system are in the interval $] \epsilon, 1]$, where $\epsilon>0$ is the smallest concentration.

$\left.\left.H_{3}\right)-i i\right)$ For $1 \leq i \leq n_{1}-1=f-2$

$$
\left\{\begin{aligned}
f_{1}^{1} & =\frac{V}{H_{1}}\left(y_{2}-x_{1}^{1}\right) \\
f_{i}^{1} & =\frac{V}{H_{i}}\left(y_{i+1}-y_{i}\right)+\frac{L}{H_{i}}\left(x_{i-1}^{1}-x_{i}^{1}\right) \\
y_{i} & =\frac{\alpha x_{i}^{1}}{1+(\alpha-1) x_{i}^{1}}
\end{aligned}\right.
$$

hence for $1 \leq i \leq n_{1}-1=f-2$ we have:

$$
\frac{\partial f_{i}^{1}}{\partial x_{i+1}^{1}}=\frac{V}{H_{i}} \frac{\partial y_{i+1}}{\partial x_{i+1}^{1}}=\frac{V}{H_{i}} \frac{\alpha}{\left(1+(\alpha-1) x_{i}^{1}\right)^{2}} \geq \frac{V}{H_{i}} \frac{\alpha}{(1+(\alpha-1) \varepsilon)^{2}}>0
$$

Similarly, for $1 \leq i \leq n_{2}-1=n-f+1$ we have:

$$
\left\{\begin{array}{l}
f_{1}^{2}=\frac{1}{H_{n}}\left[(F+L)\left(x_{2}^{2}-x_{1}^{2}\right)+V\left(x_{1}^{2}-y_{n}\right)\right] \\
f_{i}^{2}=\frac{1}{H_{i}}\left[(F+L)\left(x_{i-1}^{2}-x_{i}^{2}\right)+V\left(y_{i+1}-y_{i}\right)\right] \\
f_{n_{2}-1}^{2}=F\left(Z_{f}-x_{f}\right)+L\left(x_{n_{2}-2}^{2}-x_{n_{2}-1}^{2}\right)+V\left(y_{f+1}-y_{f}\right)
\end{array}\right.
$$

then,

$$
\left\{\begin{array}{l}
\frac{\partial f_{i}^{2}}{\partial x_{i+1}^{2}}=\frac{(F+L)}{H_{i}}>0 ; \quad 1 \leq i \leq n_{2}-2=n-f \\
\frac{\partial f_{n_{2}-1}^{2}}{\partial x_{n_{2}}^{2}}=\frac{F}{H_{f}}>0 ; \text { for } i=n_{2}-1
\end{array}\right.
$$

Simulation results: In order to show the performance of the proposed observer, we consider the binary methanol-ethanol distillation column with the following characteristics:

$$
n=12 ; f=8 ; F=1.65(\mathrm{~mol} / \mathrm{min}) ; \alpha=1.65
$$




$$
\begin{gathered}
L=4.69(\mathrm{~mol} / \mathrm{min}) ; V=5.94(\mathrm{~mol} / \mathrm{min}) ; H_{1}=5.5 \mathrm{~mol} ; H_{12}=5.5 \mathrm{~mol} \\
H_{i}=0.55 \mathrm{~mol} \text { for } 2 \leq i \leq n-1
\end{gathered}
$$

The feed composition $Z_{f}$ varies as a no uniform square signal from 0.75 to $0.38(\varepsilon(t)=0)$. The model simulation was performed under the following initial conditions:

$$
\begin{aligned}
& x(0)=(\quad 0.93000585,0.88953536,0.84223977,0.78925097 \text {, } \\
& 0.73261728,0.67505746,0.61947090,0.56837712,0.47000139 \text {, } \\
& 0.36710020,0.27017424,0.18748224,0.75)^{T}
\end{aligned}
$$

The gain of the observer was synthesized following the procedure described in proofs of the above lemma 1 with:

$$
\begin{gathered}
r_{1}=r_{2}=1, \\
\delta_{1}=1, \delta_{2}=\frac{2 n_{1}}{2 n_{2}-1} \delta_{1}=\frac{14}{11}
\end{gathered}
$$

and

$$
\begin{gathered}
S_{n_{1}}=\left[\begin{array}{ccccccc}
1.5 & -0.5 & 0 & 0 & 0 & 0 & 0 \\
-0.5 & 2 & -1.5 & 0 & 0 & 0 & 0 \\
0 & -1.5 & 4 & -2 & 0 & 0 & 0 \\
0 & 0 & -2 & 8 & -3 & 0 & 0 \\
0 & 0 & 0 & -3 & 10.5 & -4 & 0 \\
0 & 0 & 0 & 0 & -4 & 15.5 & -5 \\
0 & 0 & 0 & 0 & 0 & -5 & 17.5
\end{array}\right], \\
S_{n_{2}}=\left[\begin{array}{ccccccc}
1.5 & -0.5 & 0 & 0 & 0 & 0 \\
-0.5 & 2 & -1.5 & 0 & 0 & 0 \\
0 & -1.5 & 4 & -2 & 0 & 0 \\
0 & 0 & -2 & 8 & -3 & 0 \\
0 & 0 & 0 & -3 & 10.5 & -4 \\
0 & 0 & 0 & 0 & -4 & 15.5
\end{array}\right]
\end{gathered}
$$

then,

$$
S_{n_{1}}^{-1} C_{n_{1}}^{T}=[0.7610,0.2830,0.1237,0.0352,0.0113,0.0032,0.0009]^{T}
$$

and

$$
S_{n_{2}}^{-1} C_{n_{2}}^{T}=[0.7610,0.2830,0.1237,0.0352,0.0113,0.0032]^{T}
$$

Since the obtained results are quite similar and in order to avoid curves redundancy, we only present here those related to three trays which respectively correspond to a tray (tray 3 ) in the rectifying section, to the feed tray (composition in feed tray $x_{f}$ and feed composition $Z_{f}$ ) and finally to a tray 10 in the stripping section.

Two sets of simulation results of the observer are presented:

The first one is with free noisy outputs measurements $x_{1}(t)$ and $x_{12}(t)$ issued from simulation of model (33)(see Fig. 1). The second sets of simulation is with noisy outputs measurements $x_{1}(t)$ and $x_{12}(t)$ issued from simulation of model (33) and respectively corrupted by an adding a Gaussian noise with zero mean and an amplitude equivalent to $5 \%$ of the corresponding values (see Fig. 2).

As previously mentioned, the observer convergence can be enhanced with large values of $\theta$. However, such values are to be avoided, since the observer generate the so-called peak phenomena (see Fig. 3.) and my become noise sensitive (see Fig. 4.). To obtain good results, the choice a value of $\theta$ is one which provided a best compromise between the fast 
convergence and the noise rejection (see Fig. 5. and Fig. 6.).

\section{CONCLUSION}

In this paper, we have extended the high gain observer proposed in ${ }^{5,6}$ to a class of multioutput nonlinear system which are not necessarily control affine.

A new algorithm permitting to compute the gain of the observer is proposed. The construction is based on a symmetric positive definite matrix having a simple structure. Moreover, this S.P.D. matrix plays a key role in the proof of the convergence of the estimator.

The extension of this result to a multi-output systems (more then two outputs), requires an adequate structure. A interesting structure consists of defining a class of non affine systems extending the class of uniformly observable systems which are proposed in ${ }^{2}$.

\section{REFERENCES}

1. Besancon G., and Hammouri H., 'On uniform observation of non uniformly observable systems', systems and control leters, 29, 9-19(1996).

2. Bornard G., and Hammouri H., 'A high gain observer for a class of uniformly observable systems', Proc. 30th IEEE Conference on Decision and Control, 122 (1991), Brighton, England.

3. Deza F., 'Contribution to the synthesis of exponential observers', PhD thesis, INSA Rouen, France, (1991).

4. Gauthier J.P., and Bornard G., 'Observability for any $\mathrm{u}(\mathrm{t})$ of a class of nonlinear systems', IEEE Trans. on Aut. Control. 26 922-926(1981).

5. Gauthier J.P., Hammouri H., and Othman S., 'A simple observer for nonlinear systemsApplication to bioreactors', IEEE Trans. on Aut. Control. 37, No. 6, 875-880(1992).

6. Gauthier J.P. and Kupka I.A.K., 'Observability and observers for nonlinear systems', SIAM J. Control optim., 32,975-994(1994).

7. Hermann R. and Krenner A.J., 'Nonlinear Controllability and Observability', IEEE trans. on Aut. Control, AC-22, No. 5,728-740(1977).

8. Krener A.J. and Isidori A., 'Linearization by output injection and nonlinear observers', Syst. and Control Letters, 3, 47-52(1983).

9. Sussman H.J., 'Single-Input Observability of Continuous-Time systems', Math. System Theory, 12, 371-393(1979).

10. Xia X.H., and Gao W.B., 'Nonlinear observer design by observer error linearization', SIAM J. Control and Opt., 27, No. 1, 199-216(1989). 


\section{FIGURES CAPTION}

Fig. 1. Simulated free noisy outputs $x_{1}(t)$ and $x_{12}(t)$

Fig. 2. Simulated noisy outputs $x_{1}(t)$ and $x_{12}(t)$

Fig. 3. Comparison of estimated and simulated data from free noisy outputs (peak phenomena $\theta=4.8)$

Fig. 4. Comparison of estimated and simulated data from noisy outputs (noisy sensitive $\theta=4.2$ )

Fig. 5. Comparison of estimated and simulated data from free noisy outputs (good results $\theta=3.5$ )

Fig. 6. Comparison of estimated and simulated data from noisy outputs (good results $\theta=3.5$ ) 

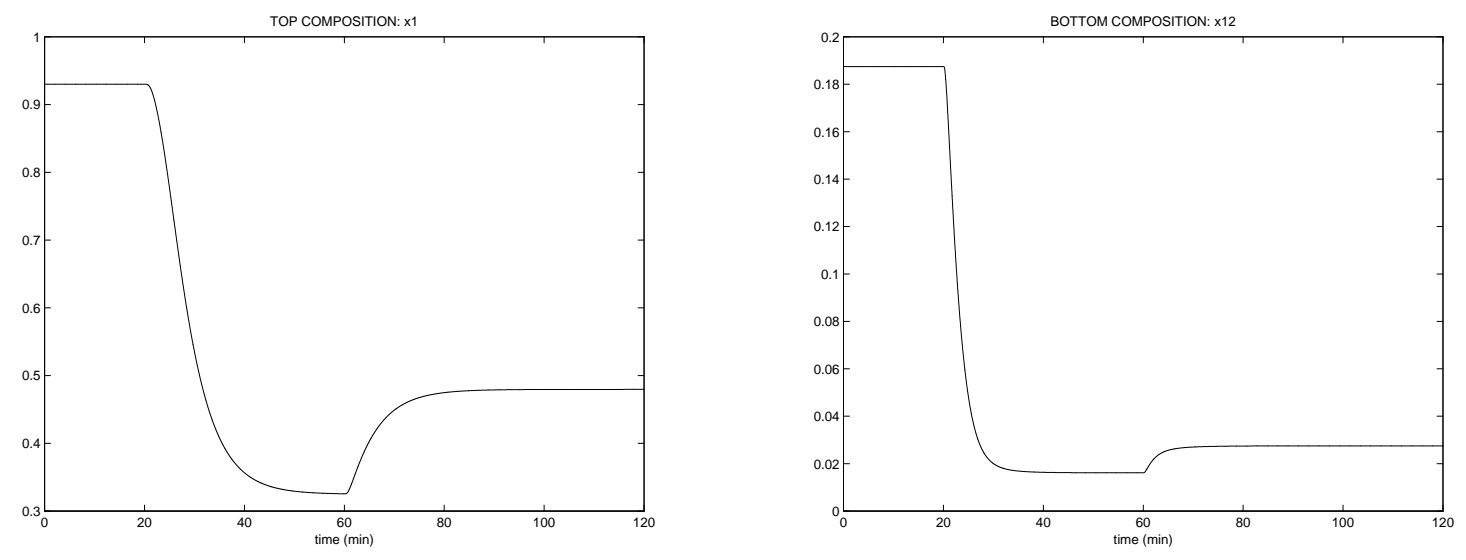

Fig. 1. Simulated free noisy outputs $x_{1}(t)$ and $x_{12}(t)$ 

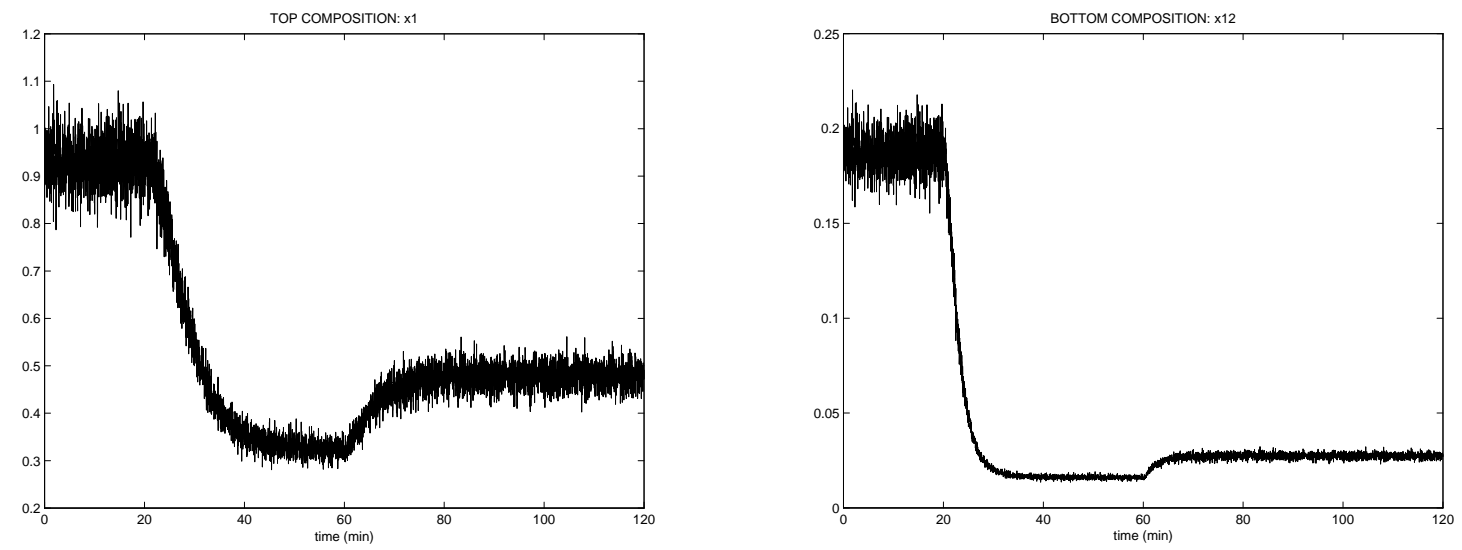

Fig. 2. Simulated noisy outputs $x_{1}(t)$ and $x_{12}(t)$ 

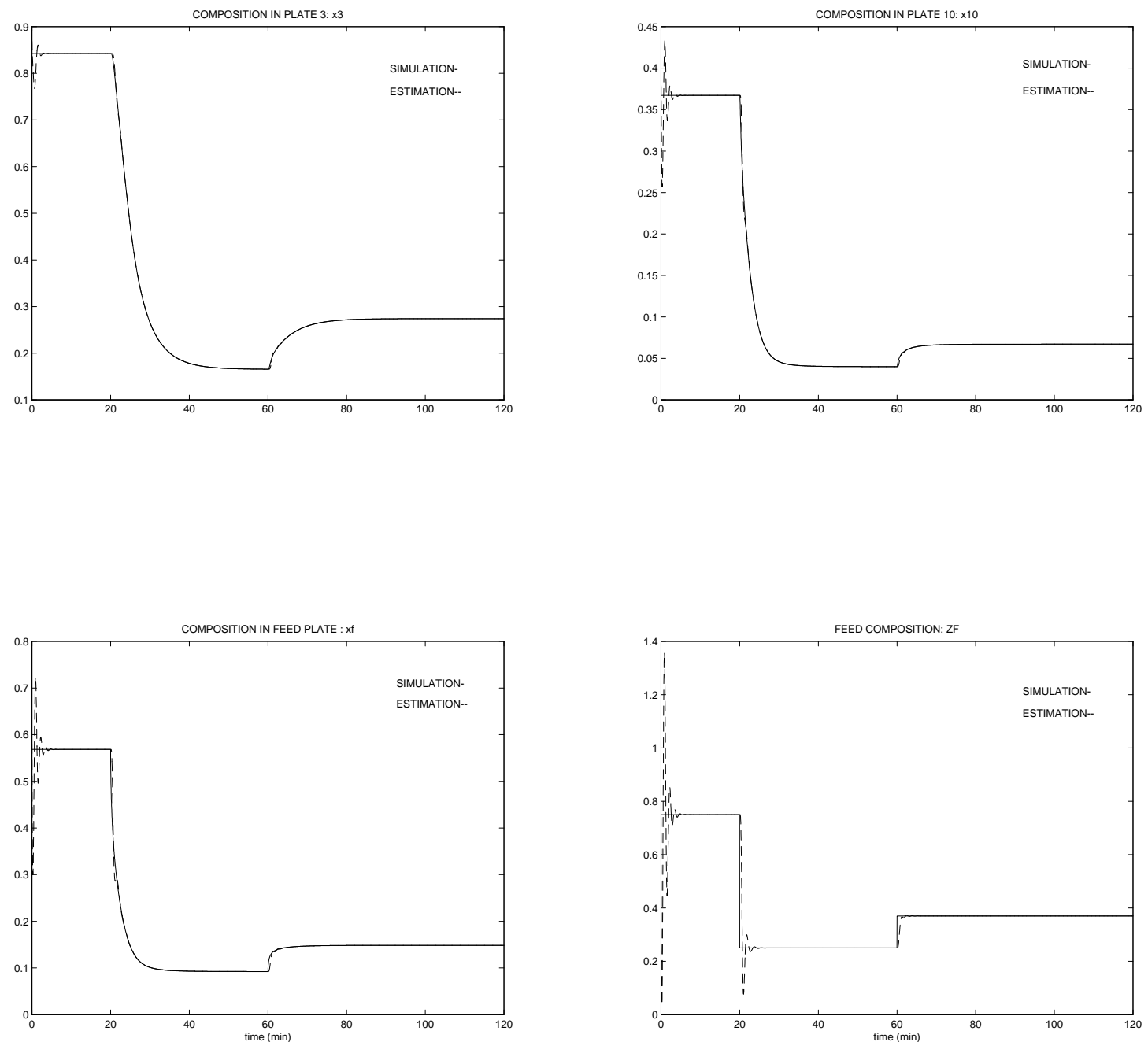

Fig. 3. Comparison of estimated and simulated data from free noisy outputs (peak phenomena $\theta=4.8$ ) 

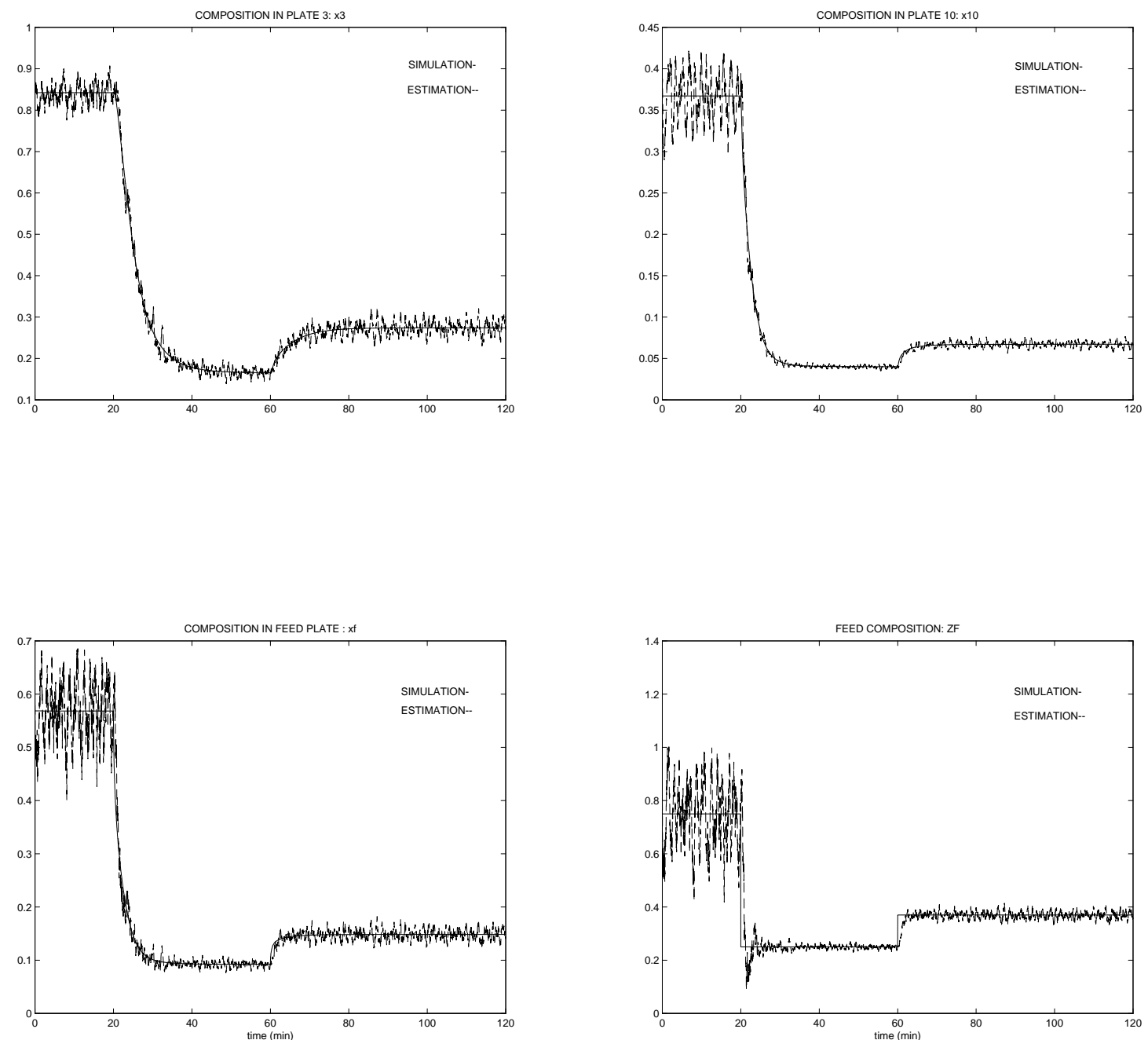

Fig. 4. Comparison of estimated and simulated data from noisy outputs (noisy sensitive $\theta=4.2$ ) 

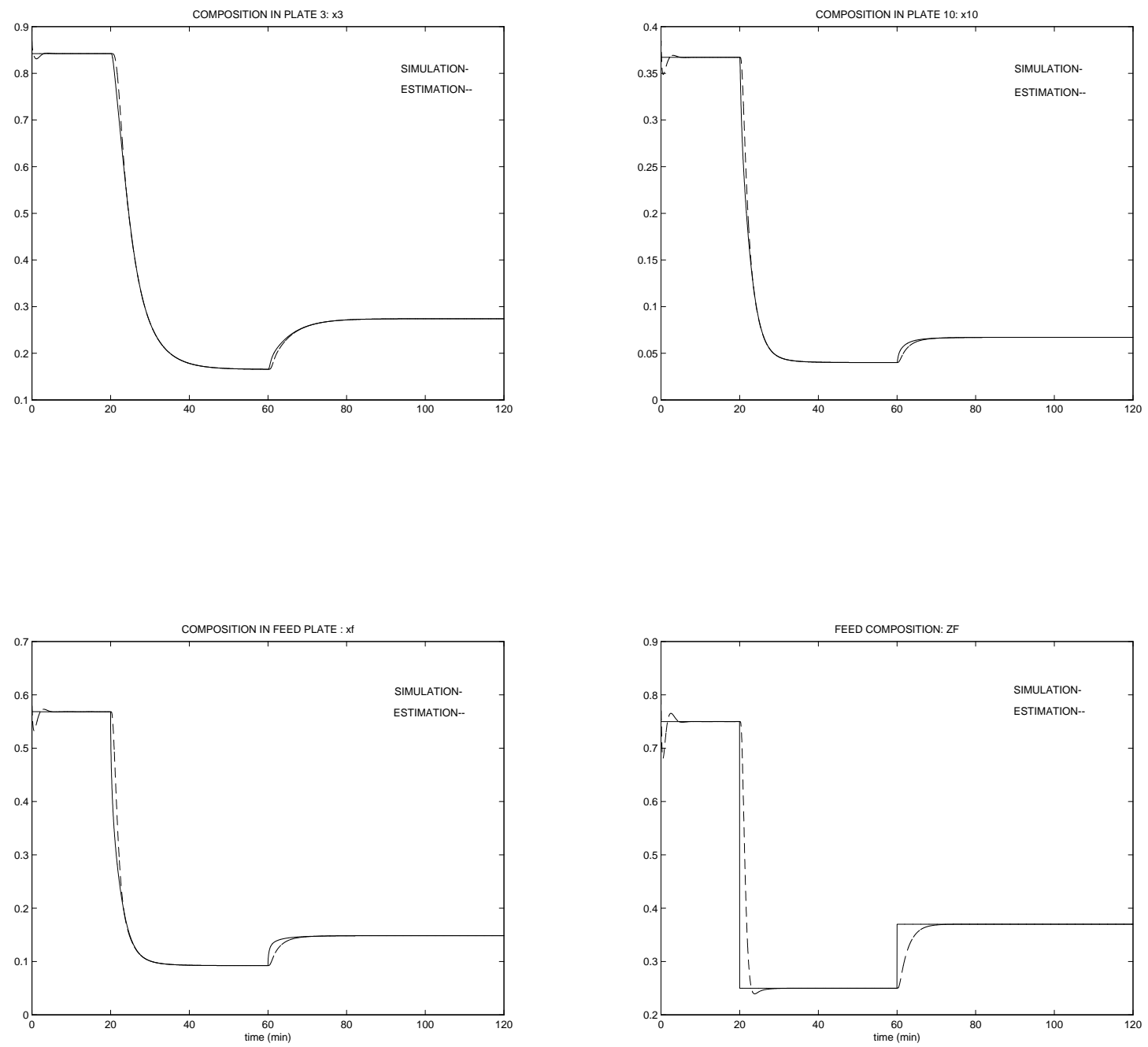

Fig. 5. Comparison of estimated and simulated data from free noisy outputs (good results $\theta=3.5$ ) 

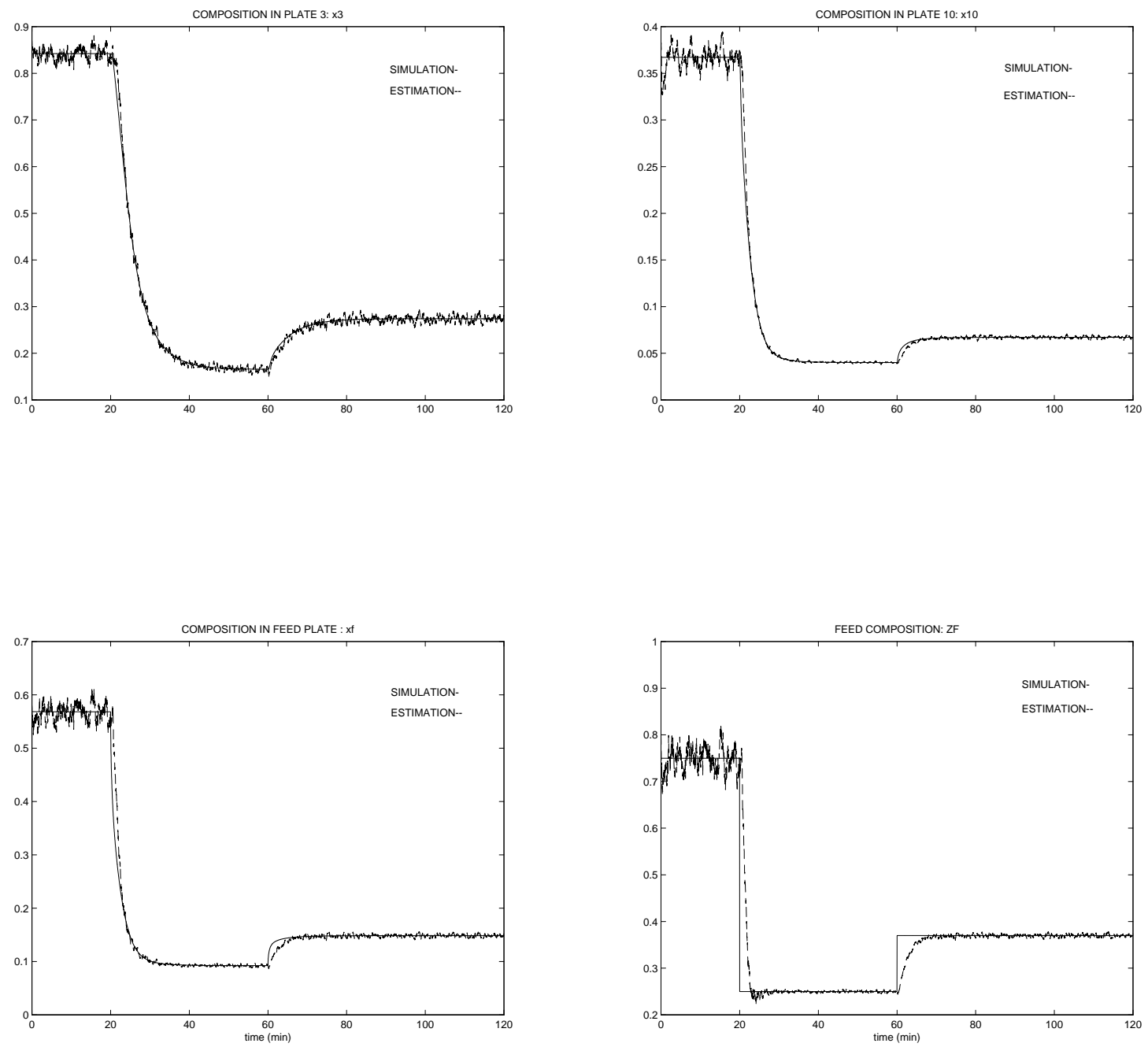

Fig. 6. Comparison of estimated and simulated data from noisy outputs (good results $\theta=3.5$ ) 